\title{
Preclinical and Clinical Studies of p38a MAP kinase inhibition to Treat Basal Forebrain Cholinergic Dysfunction and Degeneration
}

John Alam ( $\sim$ jalam@eippharma.com )

EIP Pharma Inc https://orcid.org/0000-0002-1285-1115

\section{Ralph Nixon}

Nathan Kline Institute for Psychiatric Research

Ying Jiang

Nathan Kline Institute for Psychiatric Research

Stephen Gomperts

Massachusetts General Hospital

\section{Paul Maruff}

University of Melbourne https://orcid.org/0000-0002-6947-9537

\section{Afina Lemstra}

Amsterdam University Medical Centers, Location VUmc

\section{Ursula Germann}

EIP Pharma Inc

\section{Philip Stavrides}

Nathan Kline Institute for Psychiatric Research

\section{Sandipkumar Darji}

Nathan Kline Institute for Psychiatric Research

\section{Sandeep Malampati}

Nathan Kline Institute for Psychiatric Research

James Peddy

Nathan Kline Institute for Psychiatric Research

\section{Cynthia Bleiwas}

Nathan Kline Institute for Psychiatric Research

\section{Monika Plawik}

Nathan Kline Institute for Psychiatric Research

Anna Pensalfini

Nathan Kline Institute for Psychiatric Research

\section{Dun-Sheng Yang}

Nathan Kline Institute for Psychiatric Research 
Nathan Kline Institute for Psychiatric Research

\section{Balapal Basavarajappa}

Nathan Kline Institute for Psychiatric Research

\section{John Smiley}

Nathan Kline Institute for Psychiatric Research

\section{Amanda Gardner}

EIP Pharma Inc.

\section{Kelly Blackburn}

EIP Pharma Inc.

\section{Niels Prins}

VU University Medical Center

\section{Charlotte Teunissen}

John Harrison

https://orcid.org/0000-0002-0225-4923

\section{Philip Scheltens}

Alzheimer Center Amsterdam, Department of Neurology, Amsterdam Neuroscience, Vrije Universiteit Amsterdam, Amsterdam UMC, the Netherlands

\section{Article}

Keywords: basal forebrain cholinergic neurons, Rab5, p38a kinase inhibitor

Posted Date: November 17th, 2021

DOI: https://doi.org/10.21203/rs.3.rs-1061364/v1

License: (c) (i) This work is licensed under a Creative Commons Attribution 4.0 International License. Read Full License

Version of Record: A version of this preprint was published at Nature Communications on September 21st, 2022. See the published version at https://doi.org/10.1038/s41467-022-32944-3. 


\section{Title: Preclinical and Clinical Studies of p38 $\alpha$ MAP kinase inhibition to Treat Basal Forebrain Cholinergic Dysfunction and Degeneration}

Ying Jiang ${ }^{1,2,11}$, John J. Alam ${ }^{3,11}$, Stephen N. Gomperts ${ }^{4}$ Paul Maruff 5 , Afina W. Lemstra $^{6,7}$, Ursula A. Germann ${ }^{3}$, Philip Stavrides ${ }^{1}$, Sandipkumar Darji ${ }^{1}$, Sandeep Malampati ${ }^{1}$, James Peddy ${ }^{1}$, Cynthia Bleiwas ${ }^{1}$, Monika Pawlik ${ }^{1,2}$, Anna Pensalfini, ${ }^{1,2}$, Dun-Sheng Yang ${ }^{1,2}$, Shivakumar Subbanna ${ }^{1}$, Balapal S. Basavarajappa ${ }^{1,2,8}$, John F. Smiley $^{1}$, Amanda Gardner ${ }^{3}$, Kelly Blackburn ${ }^{3}$, Hui-May Chu ${ }^{9}$, Niels D. Prins ${ }^{6,7}$, Charlotte Teunissen ${ }^{6}$, John E. Harrison ${ }^{6,10}$, Philip Scheltens ${ }^{6}$, and Ralph A. Nixon ${ }^{1,2}$

1. Center for Dementia Research, Nathan S. Kline Institute for Psychiatric Research, Orangeburg, NY, USA

2. Departments of Cell Biology and Psychiatry, NYU Langone Medical Center, NYU Neuroscience Institute, New York, NY, USA

3. EIP Pharma Inc, Boston, MA, USA

4. Massachusetts Alzheimer's Disease Research Center, Department of Neurology, Massachusetts General Hospital, Charlestown, MA, USA

5. Cogstate Ltd, London, United Kingdom

6. Alzheimer Center Amsterdam, Department of Neurology, Amsterdam Neuroscience, Vrije Universiteit Amsterdam, Amsterdam UMC, Amsterdam, The Netherlands

7. Brain Research Center, Amsterdam, The Netherlands

8. Molecular Imaging and Neuropathology Area, New York State Psychiatric Institute; Department of Psychiatry, Columbia University, New York, NY, United States.

9. Anoixis Corporation, Natick, MA, USA

10. Metis Cognition Ltd, Kilmington, United Kingdom

11. These authors contributed equally

Contact Person(s)

Ralph A Nixon, MD, PhD, Nathan S. Kline Institute for Psychiatric Research, 140 Old Orangeburg Road, Orangeburg, NY, 10962, USA P: 845-398-5423; F: 845-398-5422, ralph.nixon@nki.rfmh.org

John J. Alam, MD, EIP Pharma Inc, 120 St. James Ave, Boston, MA, 02116, USA P: 617-7444400, jalam@eippharma.com 


\section{ABSTRACT}

The endosome-associated protein Rab5 is a central player in the molecular mechanisms leading to degeneration of basal forebrain cholinergic neurons (BFCN), a long-standing target for drug development. As p38a kinase is a Rab-5 activator, we hypothesized that inhibition of this kinase held potential as an approach to treat diseases associated with BFCN loss. Herein we report that treatment with an oral small molecule p38 $\alpha$ kinase inhibitor reversed pathological disease progression in the basal forebrain in a mouse model that develops BFCN degeneration. Further, the preclinical results were successfully translated to the clinic, with improvement of clinical outcomes associated with cholinergic function in a clinical study in dementia with Lewy bodies (DLB), a disease in which BFCN dysfunction and degeneration is the primary driver of disease expression. The findings both advances a novel approach to treating DLB and validates the translational platform that provided the mechanistic rationale for advancing that approach. 
Degeneration of the basal forebrain, the primary source of cholinergic innervation in the brain, occurs in aging- and neurodegenerative disease-related cognitive disorders, including in Alzheimer's disease (AD) where basal forebrain cholinergic neuron (BFCN) dysfunction may be a major driver of the neurodegenerative process elsewhere, including in the hippocampus ${ }^{1-3}$. Further, recent publications demonstrate that BFCN loss underpins the gait dysfunction in Parkinson's disease (PD), suggesting that therapeutically targeting the cholinergic system could also address certain motor aspects of neurodegenerative disease ${ }^{4,50-51}$. Recent evidence also indicates that correcting the functional deficit in the cholinergic system, thus amplifying the physiologic release of acetylcholine is likely to be more effective than the conventional approach of compensating for BFCN dysfunction by delaying clearance of released acetylcholine with cholinesterase inhibitors 5 .

A critical pathogenic event in development of BFCN dysfunction and degeneration is impaired nerve growth factor (NGF) signaling, depriving cholinergic neurons of the neurotrophic support necessary for proper functioning and survival ${ }^{6,14}$. NGF signaling is transduced by endocytosis and retrograde trafficking of a maturing Rab5-"signaling endosome" containing the NGF receptor, TrkA, to initiate a transcriptional program. Cholinergic neurons, with long axonal projections throughout the cortex, are particularly vulnerable to disruption of this retrograde signaling process from distant synaptic connections back to the cell body. From a pathogenic mechanistic standpoint, the protein Rab5, a GTPase and master signaling molecule regulating endocytosis and endosome function, is implicated in development of impaired NGF signaling. Both Rab5 hyper-activation and lowered endosome recycling rates impair NGF signaling, partly due to enlargement of endosomes which slows their retrograde transport and trophic signaling ${ }^{7,8}$, leading to cholinergic atrophy in Down syndrome (DS) mouse models ${ }^{7,9-11}$. These DS models 
essentially recapitulate adult-onset basal forebrain cholinergic neurodegeneration ${ }^{12}$. Further, Rab5 hyperactivation in vivo in Rab5 overexpressing transgenic mice causes BFCN degeneration ${ }^{12}$. These findings establish Rab5 as a therapeutic target for BFCN dysfunction. Moreover, results from animal studies also indicate that the neurodegeneration of BFCNs is reversible, as the BFCNs do not die with age and disease; rather, they lose cholinergic phenotype and functional properties, and this loss can be reversed by direct NGF infusion to the basal forebrain ${ }^{13,14}$. Thus, pharmacologically restoring NGF signaling through targeting Rab5 has the potential to reverse disease progression by increasing numbers of functional cholinergic neurons.

As the alpha isoform of $\mathrm{p} 38 \alpha$ kinase is a major Rab5 regulator and activator ${ }^{15}$, and it has been implicated in the regulation of retrograde axonal transport ${ }^{16}$, we hypothesized that $\mathrm{p} 38 \alpha$ inhibition would be a pharmacological approach to treating diseases associated with BFCN dysfunction. Accordingly, we evaluated a specific p38 $\alpha$ kinase inhibitor neflamapimod $(\mathrm{NFMD})^{17-19}$, preclinically in a DS mouse model (Ts2 mouse) $)^{12}$ that demonstrates BFCN degeneration and, subsequently, clinically in a placebo-controlled phase 2 a study in patients with dementia with Lewy bodies (DLB), where BFCN dysfunction and degeneration is considered to be the primary driver of disease expression and progression ${ }^{20-22}$. Herein we report successful translation of the scientific understanding of mechanisms of cholinergic degeneration to beneficial effects on BFCNs both preclinically and clinically, with neflamapimod reversing pathological disease progression in the basal forebrain in the preclinical study and improving clinical outcomes associated with cholinergic function in the clinical study in DLB patients. 


\section{RESULTS}

Neflamapimod treatment of $T_{s} 2$ mice reverses endosomal pathology and restores number of cholinergic neurons

Ts2 mice, a DS mouse model that develops adult-onset Rab5+ endosomal pathology and cholinergic degeneration in the basal forebrain ${ }^{12}$, and control $2 \mathrm{~N}$ (wild type) mice were treated at approximately six months of age with either neflamapimod $(3 \mathrm{mg} / \mathrm{kg}$ body weight, twice daily (BID)) or vehicle (1\% Pluronic F108) by oral gavage for 28 days. The number and size of Rab5GTP-positive endosomes, determined by Image J analysis, were greater in Ts2 than in wildtype mice, while, with neflamapimod treatment, both parameters were normalized to values seen in the wildtype mice (Fig. 1A and B). Similarly, the intensity ratio of Rab5-GTP (activated form of rab5) to total Rab5 was significantly higher in vehicle-treated Ts2 than in wildtype mice (Fig. 1C) collectively and reduced with neflamapimod treatment in Ts2 mice and not in wildtype mice; results which suggest neflamapimod impacts aberrant Rab5 activation, without impacting physiologic Rab5 activation. In Ts2 mice, basal forebrain neurodegeneration is well established by 6 to 7 months of age ${ }^{12}$ and the number of medial septal choline acetyltransferase-positive $(\mathrm{ChAT}+)$ neurons (i.e., cholinergic neurons) continues to decline as the mice age (Fig. 1D). Neflamapimod-treated Ts2 mice had significantly higher numbers of ChAT+ neurons at the end of the four-week treatment period than did vehicle-treated Ts2 mice $(p<0.005)$, exhibiting numbers comparable to those in the wildtype mice (Fig. 1E,F). Notably, in vehicle-treated Ts2 mice, ChAT + neurons had an abnormal morphology, with neurite swelling (yellow arrows) or atrophy (orange arrow), perikaryal atrophy (red arrows), and below normal ChAT immunoreactivity (green arrows) (Fig. 1H), while neflamapimod treatment appeared to normalize their morphology (Fig. 1G,H). 
Synaptic function, as represented by the long-term potentiation (LTP) of synaptic transmission in the Schaffer collateral synapses (CA3-CA1) of Ts2 mice, was improved modestly $(p<0.05,18 \%$ increase in fEPSP slope at 110 minutes) by neflamapimod treatment (Figures 2A, B and C). Behavioral evaluations considered dependent on basal forebrain cholinergic function, novel object recognition (NOR) and open field tests, were examined in a separate cohort of animals. Because of limited availability of Ts2 mice at the time, no vehicleadministered animals could be included in these evaluations. Instead, the behavioral tests were performed both pre- and post- four weeks dosing with neflamapimod and, in addition, wild type mice were included for comparison. For both the NOR and open field tests, there was a significant difference between wild-type and Ts2 mice pretreatment that was not apparent at the end of neflamapimod treatment (Figs. 2D and E). For the open field test, the performance at the end of treatment also represented a significant improvement from performance at the pretreatment baseline (Figure 2E).

The positive effects of neflamapimod on pathology and function in Ts 2 mice appear to be mediated by inhibition of the p38a kinase pathway

Further biochemical analyses were conducted to understand the underlying mechanisms. First, p38, phosphorylated-p38 (p-p38), and its downstream substrates MK2 $2^{23,24}$ and MNK124,25 were assessed in tissue homogenates of the brain cortex by Western blotting, as described previously $^{12}$. Indicating target engagement, neflamapimod treatment normalized p-p38 levels, while significantly decreasing levels of total MK2 and MNK1, as well as pMNK1, in Ts2 mice (Figs. 3A and B).

As reduction of $\mathrm{p} 38 \alpha$ activity in APP/PS1 double transgenic mice leads to decreased BACE1 protein expression ${ }^{26}$, BACE1 protein levels were similarly evaluated in the brain cortex 
samples. Ts2 mice treated with neflamapimod, compared with vehicle, had significantly lower BACE1 protein levels (Figs. 3C and D; $p<0.05$ for neflamapimod $v s$. vehicle-treated Ts2 mice). In addition, levels of $\beta C T F$, the product of APP cleavage by BACE1, which were significantly higher in Ts 2 than in wildtype mice $(p<0.001)$, were decreased significantly by neflamapimod treatment in the Ts2 mice (Figs. $3 \mathrm{C}$ and $\mathrm{D}, p<0.001$ ).

Neflamapimod treatment in patients with DLB improves cognition within domains associated with cholinergic function

The AscenD-LB study (NCT04001517) was a 91-patient, 16-week, double-blind placebo-controlled phase 2a clinical study in patients with mild-to-moderate DLB by consensus clinical criteria ${ }^{27}$ including demonstrated abnormality in dopamine uptake by DaTscan ${ }^{\mathrm{TM}}$ (Ioflupane I123 SPECT), receiving cholinesterase inhibitor therapy ( $>3$ months, stable dose $>6$ weeks). The aim of this exploratory study was to evaluate the cognitive effects of neflamapimod in this patient population, utilizing a six-test neuropsychological test battery (NTB) designed to assess attention and executive function. No formal sample size calculations were performed but based on prior experience with use of a NTB, 40 participants per arm was considered sufficient for the primary objective. Patients were randomized 1:1 to either neflamapimod 40mg capsules or matching placebo; and then, based on body weight, assigned to either a twice-daily (BID) [weight $<80 \mathrm{~kg}$; 40mg BID (80mg/day) neflamapimod or placebo BID) or thrice-daily (TID) [weight $\geq 80 \mathrm{~kg}$; 40mg TID (120mg/day) neflamapimod or placebo TID] regimen. The weightbased dosing regimen was utilized because pharmacokinetic data available at the time of study start indicated that 40mg BID would achieve the targeted 12-hour plasma drug exposure of 100 $\mathrm{ng} * \mathrm{hr} / \mathrm{mL}$, but only in patients weighing less than $80 \mathrm{~kg}$ (thus requiring $40 \mathrm{mg}$ TID in the higher weight range). However, during the study, additional data became available, including from a 
study in $\mathrm{AD}^{20}$, indicating that $40 \mathrm{mg} \mathrm{BID}$ would not achieve the target plasma drug exposure and would likely be ineffective. Indeed, measured plasma drug concentrations in the current study were 50\% higher in 40mg TID recipients [median steady-state concentration ( $\left.\mathrm{C}_{\mathrm{SS}}\right)$ of 10.2 $\mathrm{ng} / \mathrm{mL}$ ] than in 40mg BID recipients (median $\mathrm{C}_{\mathrm{SS}}=6.8 \mathrm{ng} / \mathrm{mL}$ ). As a result, the efficacy endpoints were analyzed in two ways, (1) considering all neflamapimod recipients as one dose group as originally intended (an evaluation in which treatment effects are diluted by 40mg BID not achieving target plasma drug concentrations) and (2) considering 40mg TID recipients separately. As this was an exploratory study, p-values were not adjusted for multiple comparisons. There were no significant differences in baseline disease characteristics between groups, either comparing all neflamapimod recipients vs. placebo, or 40mg TID vs. either placebo or placebo TID (Supplemental Table 1; Supplemental Figure 1 shows Consort Flow diagram).

The mean NTB composite $z$-score, in which the individual tests are weighted equally, over the course of study is shown in Figure 4A. In the placebo group, this cognitive composite remained stable or worsened slightly over the 16 weeks of the study, indicating no placebo or learning effects in the data. For the 40mg BID group, the cognitive composite results were very similar to those for the placebo group while, beginning at week 4 and maintained to week 16 , there was an apparent improvement in the 40mg TID group. With neflamapimod 40mg BID being similar to placebo, there were no significant differences $(p>0.2)$ when the combined neflamapimod groups were compared with placebo. For the 40mg TID group, the cognitive composite improved from baseline and was significantly different over the course of the study from either placebo ( $p=0.049$, mean difference $=0.18$ 95\%CI:0.00-0.35, Cohen's d effect size $(\mathrm{ES})=0.47)$ or placebo TID $(p=0.049$, difference $=+0.11,95 \%$ CI $0.00-0.21, \mathrm{ES}=0.49)$. 
As attention is the cognitive domain regarded as most specific for the cholinergic system, performance on the two NTB tests that measure attention and processing speed (i.e., the Cogstate Detection and Identification Tests) were combined to yield an Attention Composite (Figure 4B). In this analysis there was a significant difference favoring 40mg TID over placebo $(p=0.023$, difference $=0.278,95 \% \mathrm{CI} 0.041-0.515, \mathrm{ES}=0.41)$ and a trend favoring neflamapimod-treatment in the comparison of the two higher weight cohorts, $40 \mathrm{mg}$ TID vs. placebo TID ( $p=0.099$, difference $=0.253,95 \% \mathrm{CI}:-0.004-0.511, \mathrm{ES}=0.33$ ).

Neflamapimod treatment did not impact the secondary cognitive endpoint considered most strongly depend on hippocampal function, verbal list learning (measured using the International Shopping List Test, Supplemental Figure 2).

Neflamapimod treatment in patients with DLB improved a global measure of cognition and function, as well as functional mobility

The Clinical Dementia Rating Scale Sum of Boxes (CDR-SB) scale, designed to assess both cognition and function, scores six domains (memory, orientation, judgement $\&$ problem solving, community affairs, home $\&$ hobbies, and personal care) on a $0-3$ scale (total range $0-18$, higher scores indicating worse dementia). The CDR-SB was performed at baseline, week 8 , and week 16. On-study, the CDR-SB scores increased (worsened) in the placebo group, while there was less worsening in the neflamapimod treated patients (Figure 4C). Comparing all neflamapimod with all placebo, there was significant improvement with neflamapimod treatment $(p=0.023$, difference $=-0.45,95 \%$ CI:-0.83,-0.06;ES=0.31). Compared with the all-neflamapimod vs. all-placebo analysis, the difference between neflamapimod treatment and placebo was greater in the 40mg TID $v$ s. placebo TID analysis ( $\mathrm{p}=0.005$, difference $=-0.63,95 \% \mathrm{CI}:-1.06$,- 
$0.21 ; \mathrm{ES}=0.31)$ and in the $40 \mathrm{mg}$ TID $v s$. all placebo analysis $(\mathrm{p}=0.007$, difference $=-0.56,95 \% \mathrm{CI}$ :$0.96,-0.16 ; \mathrm{ES}=0.35)$.

Gait dysfunction due to parkinsonism is very common in patients with DLB and recent evidence indicates that gait dysfunction is to great extent due to deficits in basal forebrain cholinergic function ${ }^{4}$. Gait in the AscenD-LB study was assessed with the Timed Up and Go (TUG) Test (Fig. 4D), measuring functional mobility by monitoring the time that a subject takes to rise from a chair, walk three meters, turn around 180 degrees, walk back to the chair, and sit down while turning 180 degrees. In the comparison of the combined neflamapimod groups (i.e., "all neflamapimod" participants) to placebo, there was significant reduction in the time required to complete the TUG test (i.e., improvement) with neflamapimod treatment ( $\mathrm{p}=0.044$, difference $=-1.36 \mathrm{sec}, 95 \% \mathrm{CI}:-0.04$ to $-2.69, \mathrm{ES}=0.22$ ). The beneficial treatment effect was greater in 40mg TID patients, as the effect size was greater in the comparison of 40mg TID to either placebo ( $\mathrm{p}=0.024$, difference $=-1.40 \mathrm{sec}, 95 \% \mathrm{CI}:-0.19,-2.61 ; \mathrm{ES}=0.50)$ or placebo TID $(\mathrm{p}=0.099$, difference $=-1.43$ seconds, $95 \% \mathrm{CI}:-3.15,0.0 .28 ; \mathrm{ES}=0.44)$.

\section{Efficacy of Neflamapimod was greater in DLB than in mixed $A D-D L B$}

In patients with DLB, elevated levels of the protein phosphorylated tau (ptau) have been reported to identify the presence of Alzheimer's disease (AD) co-pathology, particularly temporal lobe tau pathology 28 ; and patients with such co-pathology are considered to have mixed AD-DLB, rather than DLB. Accordingly, plasma ptau181 levels in baseline plasma samples were quantitated utilizing the Simoa ${ }^{\circledR}$ platform in a laboratory where the plasma ptau181 cut-off for $\mathrm{AD}$ co-pathology had been set at $2.2 \mathrm{pg} / \mathrm{mL}$ (i.e., plasma ptau $181 \geq 2.2 \mathrm{pg} / \mathrm{mL}$ associated with existence of $\mathrm{AD}$ co-pathology). 
At baseline, 22 of $41(53 \%)$ of placebo and 22 of 42 (54\%) of neflamapimod participants in the efficacy analysis population had ptau $181<2.2 \mathrm{pg} / \mathrm{mL}$ (i.e., had DLB, rather than mixed AD-DLB). When the efficacy analyses were repeated, after taking into account plasma ptau 181 levels, the response in the patients with baseline plasma ptau $181<2.2 \mathrm{pg} / \mathrm{mL}$ (i.e., those predicted to not have AD co-pathology and therefore "pure DLB") appears to be better than the response in patients with baseline plasma ptau181 levels $\geq 2.2 \mathrm{pg} / \mathrm{mL}$ (i.e., those predicted to have AD co-pathology, and therefore mixed AD-DLB); see Figure 5. Further, as shown in Table 1 (lower half), when individual endpoints showing positive treatment effects in the main analysis were analyzed in only patients with pure DLB (defined by ptau181<2.2 pg/mL at baseline), the magnitude of the treatment effect was high with the statistics particularly robust for the comparison of 40mg TID vs. placebo or vs. placebo TID for attention composite, TUG and CDR-SB. For the NTB, the magnitude of the treatment effect is also greater than in the main analysis but with the smaller sample size, did not reach significance. The magnitude of the treatment effect in patients with pure DLB for the comparison between 40mg TID vs. placebo (or placebo TID), as evaluated by effect size, was substantially higher than for the full efficacy population. In addition, in the 40mg TID vs. placebo TID comparison, the effect size was consistently greater than 0.6 , the level above which is considered to be a clinically important treatment effect.

\section{Neuropsychiatric Outcomes and Safety in the Clinical Study}

Four specific domains within the 10-item Neuropsychiatric Inventory [depression (dysphoria), anxiety, hallucinations, and agitation/aggression] were secondary outcome measures in the study. Of these, the domain most specific for both DLB and the cholinergic system is hallucinations ${ }^{29}$, for which there was a significant effect favouring neflamapimod treatment for 
severity (Supplemental Figure 4; $\mathrm{p}=0.050$ for all neflamapimod vs. placebo, $\mathrm{p}=0.019$, for neflamapimod 40mg TID vs. placebo). There were no discernible treatment effects for the other three domains of the NPI-10 (Supplemental Figure 5).

Neflamapimod treatment was well tolerated with no study-drug associated treatment discontinuations or serious adverse events (SAEs) reported (See Supplemental Table 1 for all treatment discontinuations and SAEs). The only treatment-emergent adverse events occurring at an incidence $>5 \%$, in either neflamapimod- or placebo-treated patients, were falls $(13 \%$ in neflamapimod versus $9 \%$ in placebo), headache $(9 \%, 4 \%)$, diarrhea $(7 \%, 11 \%)$, nausea $(7 \%$, $7 \%)$, and tremor $(0 \%, 7 \%)$. Among neflamapimod-treated patients, the incidence $(3 / 20,15 \%$ for each) of diarrhea and headache were higher in those receiving 40mg TID than in those receiving 40mg BID (0/26, $0 \%$ for diarrhea; $1 / 26,3 \%$ for headache); the incidence of falls was higher in those receiving 40mg BID (5/26, 19\%) than in those receiving 40mg TID (1/20, 5\%). 


\section{DISCUSSION}

Consistent with our overarching hypothesis, Ts2 (DS) mice with established basal forebrain pathology, when treated with the p38 $\alpha$ inhibitor neflamapimod, showed decreased Rab5 activation, reversal of Rab5+ endosomal pathology, and, most important, restoration of the number and morphology of cholinergic neurons in the medial septal region of the basal forebrain. As with the NGF studies, we interpret the increased cholinergic neuron numbers as restoration of the cholinergic phenotype in neurons that have, otherwise, continued to survive, rather than as a neuro-regenerative effect ${ }^{13,30}$. Supporting that interpretation, p38 MAPK has been implicated in cholinergic differentiation ${ }^{31}$. Moreover, inflammation, in a p38 MAPKdependent manner, reversibly reduced ChAT expression in the basal forebrain ${ }^{32}$. Though not a main aim of the Ts2 mouse study, behavioral assessments did indicate a functional impact of increasing the number of cholinergic neurons, in that neflamapimod treatment restored performance to wildtype levels in behavioral tests linked to cholinergic function, the Open Field and the NOR tests ${ }^{33-35}$. In contrast, neflamapimod treatment had only minor effects on synaptic plasticity in the hippocampus, where the cholinergic system may not be the major determinant of the outcome as defects in hippocampal function in the DS mouse model may be independent of cholinergic transmission ${ }^{36}$ and represent fixed structural deficits, including frank neuronal $\operatorname{loss}^{37,38}$. Our functional results may also be evaluated in the context of published studies with neflamapimod in rat models, where behavioral outcomes are more readily assessed ${ }^{17}$. First, neflamapimod reversed deficits in Morris-water-maze performance ${ }^{17}$, attributed to basal forebrain dysfunction ${ }^{39}$, in aged rats. Second, neflamapimod improved functional recovery after a transient-ischemia induced stroke in rats ${ }^{59}$, with basal forebrain cholinergic function regarded as rate-limiting for the recovery process ${ }^{40,41}$. Together with these previously reported findings, 
the effects of neflamapimod in Ts2 mice provide preclinical proof-of-concept for p38 $\alpha$ inhibition to reverse BFCN dysfunction.

The preclinical mechanistic findings were consistent with our original hypothesis that p38 $\alpha$ inhibition is an approach to decrease Rab5 activation and reverse the block in endosomal trafficking and signaling. In addition, the data provide insight on additional relevant targets that may be contributing to drug activity. Specifically, neflamapimod treatment decreased Rab5-GTP levels, a marker of Rab5 activation, and reversed the Rab5+endosomal pathology that is a direct histopathological marker of the block in endosomal trafficking underlying the defect in NGF signaling in Ts 2 mice ${ }^{6,7}$. In separate experiments, restoration of cholinergic neuron numbers was observed following neflamapimod treatment in Rab5-overexpressing transgenic mouse, which otherwise develop basal forebrain cholinergic degeneration ${ }^{42}$; further supporting the activity of neflamapimod on Rab5-mediated cholinergic neuron loss. The beneficial effects were associated with p38 $\alpha$ inhibition, because neflamapimod treatment normalized levels of activated, i.e., phosphorylated p38 $\alpha$ (in addition to being activated by upstream kinases, $\mathrm{p} 38 \alpha$ autoactivates $^{43,44}$ ) and lowered levels of those of its downstream substrates MK2 and MNK1 ${ }^{45}$. Furthermore, neflamapimod treatment decreased BACE1 protein levels, consistent with reports that neuronal p38a knockout in APP/PS1 (“Alzheimer's") transgenic mice led to decreased BACE1 protein levels, through increased autophagy-lysosome mediated degradation of the BACE1 protein ${ }^{26,46,47}$. Moreover, in a recent study, p38 $\alpha$ activity decreased BACE1 levels in synaptic terminals by increasing retrograde axonal transport of BACE1 to lysosomes for degradation $^{48}$. Thus, the finding that neflamapimod decreases BACE1 protein levels in Ts 2 mice provides further evidence that the drug improves retrograde axonal transport of endosomes, the fundamental pathophysiologic defect being targeted with our therapeutic approach. Finally, the 
reduction in $\beta C T F$ levels, resulting from decreased BACE1 protein levels, is also a likely contributor to the effects on cholinergic degeneration and function ${ }^{7,12}$.

With a mechanistic foundation provided by the animal studies, the results of the clinical study provided direct evidence that the preclinical results could be translated to the clinic, with neflamapimod treatment leading to significant dose-dependent improvements in cognition, motor function and potentially visual hallucinations in a neurodegenerative disease characterized by substantial basal forebrain cholinergic loss, DLB. Despite the use of a relatively small sample in this exploratory study, the consistency of the benefits with neflamapimod 40mg TID, relative to placebo, across multiple aspects of DLB endpoints that are related to cholinergic function but are otherwise uncorrelated, makes the results of this clinical trial compelling. Further, as disease expression in the patients with "pure DLB" (rather than mixed AD-DLB) would be expected to most specifically driven by BFCN dysfunction, the specificity for, and magnitude of the clinical efficacy in this patient sub-group further supports that neflamapimod has potent activity against BFCN dysfunction. Notably, all these improvements were observed in patients already receiving cholinesterase inhibitors and the effect size on cognition was, in fact, greater for neflamapimod than that reported for rivastigmine on a similar cognitive test battery ${ }^{49}$. In addition, whereas cholinesterase inhibitors do not impact motor function ${ }^{50}$, neflamapimod demonstrated an effect on functional mobility. The beneficial effects on both cognitive and motor function may be reflected in the CDR-SB, a test which has the ability to be impacted by effects on cognition, as well as on motor function through effects on the functional domains (most obviously on personal care). Remarkably, the results align with a recent study in patients with DLB or PD undergoing deep brain stimulation of the nucleus basalis of Meynert (NBM) within the basal forebrain. That study defined three functional networks, one involved in volitional control of movement, one in 
attention and memory, and a third in control of visual function ${ }^{51}$. The effects we observed on motor function are also consistent with a series of recent clinical translational publications connecting the NBM to gait impairment in $\mathrm{PD}^{4,52,53}$. As such, while the role of the basal forebrain cholinergic system in attention and memory is well established, our clinical findings in the context of the recent literature further confirm the long-suspected role of the basal forebrain in motor learning and plasticity.

Consistent with the preclinical results, in contrast to positive effects seen on endpoints related to cholinergic function, minimal to no effects were seen in the clinical study on the cognitive endpoint most dependent on normal hippocampal function, the ISLT. This finding further supports a drug effect specifically related to the mechanism of action of neflamapimod on the cholinergic system. The relative lack of effect of neflamapimod on the hippocampus is consistent with clinical effects of cholinesterase inhibitors that, show pro-cognitive effects in AD primarily on attention ${ }^{54-57}$, i.e., an endpoint considered related to cholinergic function; while on the endpoint most specific for hippocampal function, delayed recall, cholinesterase inhibitors have shown no discernible effects ${ }^{57-58}$. At the same time, donepezil reduced the rate of hippocampal atrophy progression ${ }^{59}$, possibly through reducing the rate of atrophy progression in the basal forebrain ${ }^{60}$, a finding that is consistent with the notion that degeneration in the basal forebrain precedes, and is a major contributor to neurodegeneration and disease progression in the hippocampus in AD. Indeed, neflamapimod treatment in a 24-week placebo-controlled study in $\mathrm{AD}$, while not improving episodic memory function, did improve, relative to placebo, CSF markers of neurodegeneration and there was evidence of a reduction in progression of disease by clinical endpoints, including the episodic memory endpoint ${ }^{19}$. The combined findings suggest that treating basal forebrain cholinergic degeneration has the potential to treat disease 
progression in the hippocampus in $\mathrm{AD}$, but longer duration trials are required to evaluate that potential.

The clinical results provide a foundation for further evaluation of neflamapimod in patients with DLB, where the primary pathology is in the basal forebrain. However, a larger study is required to better define the magnitude of the benefit and only a longer duration trial can address whether neflamapimod treatment impacts DLB disease progression. Our results, combined with other clinical and preclinical data, also suggest that neflamapimod, by improving basal forebrain cholinergic function, has the potential to slow disease progression in the hippocampus in AD. Beyond implications specific to neflamapimod, our results provide validation of our pathogenic model of cholinergic degeneration and of the Ts2 DS mouse as a translational platform for target validation, drug discovery and development, and for obtaining preclinical proof-of-concept for novel therapeutic approaches to treating basal forebrain cholinergic dysfunction and degeneration. 
Table 1. Statistical Analyses of Outcome Measures Related to Cholinergic

Function in the Clinical Study

\begin{tabular}{|c|c|c|c|c|c|c|}
\hline \multicolumn{7}{|c|}{ Results in Full Efficacy Population } \\
\hline & \multicolumn{2}{|c|}{$\begin{array}{c}\text { Improvement in all NFMD } \\
\text { vs. placebo }\end{array}$} & \multicolumn{2}{|c|}{$\begin{array}{l}\text { Improvement in NFMD } \\
40 m g \text { TID vs. placebo }\end{array}$} & \multicolumn{2}{|c|}{$\begin{array}{l}\text { Improvement in NFMD } \\
40 m g \text { TID vs. placebo TID }\end{array}$} \\
\hline & p-value & Effect size & p-value & Effect size & p-value & Effect Size \\
\hline \multicolumn{7}{|l|}{ Cognition: } \\
\hline$N T B^{*}$ & $>0.2$ & 0.10 & 0.049 & 0.47 & 0.049 & 0.49 \\
\hline Attention & 0.171 & 0.18 & 0.023 & 0.41 & 0.073 & 0.33 \\
\hline$C D R-S B$ & 0.023 & 0.31 & 0.005 & 0.38 & 0.007 & 0.31 \\
\hline Timed Up and Go & 0.044 & 0.22 & 0.024 & 0.50 & 0.099 & 0.44 \\
\hline \multicolumn{7}{|c|}{ Results in Patients with Baseline Plasma ptau181 $<2.2 \mathrm{pg} / \mathrm{mL}$} \\
\hline & \multicolumn{2}{|c|}{$\begin{array}{c}\text { Improvement in all NFMD } \\
\text { vs. placebo }\end{array}$} & \multicolumn{2}{|c|}{$\begin{array}{l}\text { Improvement in NFMD } \\
40 m g \text { TID vs. placebo }\end{array}$} & \multicolumn{2}{|c|}{$\begin{array}{l}\text { Improvement in NFMD } \\
\text { 40mg TID vs. placebo TID }\end{array}$} \\
\hline & p-value & Effect size & p-value & Effect size & p-value & Effect Size \\
\hline \multicolumn{7}{|l|}{ Cognition: } \\
\hline$N T B^{*}$ & $>0.2$ & 0.15 & 0.133 & 0.56 & 0.123 & 0.61 \\
\hline Attention & 0.185 & 0.29 & 0.021 & 0.78 & 0.034 & 0.70 \\
\hline$C D R-S B$ & 0.129 & 0.58 & 0.031 & 0.74 & 0.009 & 0.98 \\
\hline Time up and Go & 0.024 & 0.40 & $<0.001$ & 0.74 & 0.010 & 0.70 \\
\hline
\end{tabular}

*NTB: 6-test Neuropsychological Test Battery evaluating attention and executive function

Note: Cohen's d effect size reported 


\section{FIGURE LEGENDS}

\section{Figure 1. Normalized Rab5+ endosome number/size and restored cholinergic neuronal numbers in Ts2 mice treated with Neflamapimod (NFMD).}

Representative images of wildtype $(2 \mathrm{~N})$, Ts2 and Neflamapimod-treated Ts2 (Ts2-NFMD)

mouse MSN regions labeled with Rab5-GTP (red) and Rab5a (green) antibodies; arrows point to the Rab5-positive neurons which are shown enlarged in the lower panel (scale bar, $15 \mu \mathrm{m}) . \mathrm{b}$. The numbers, sizes and areas of Rab5-GTP-positive endosomes were determined by Image $\mathbf{J}$ analysis ( $n=36$ neurons per treatment group), normalized to values seen in the $2 \mathrm{~N}$ mice treated with vehicle, and graphed using GraphPad Prism8. c. The ratio of Rab5-GTP to total Rab5 intensities was also determined with Image $\mathrm{J}$ and graphed using GraphPad Prism8 (n=57 neurons/each treatment group). $\mathrm{d}$. The number of stereologically counted ChAT + neurons in the MSN region of $2 \mathrm{~N}(\mathrm{n}=27)$ and Ts2 $(\mathrm{n}=28)$ mice was graphed versus age of mice (months). e. Representative images of diaminobenzidine (DAB)-stained ChAT + neurons in the MSN region of $2 \mathrm{~N}$ and Ts 2 mice treated with either vehicle or NFMD (scale bar, $100 \mu \mathrm{m}$ ). f. Quantification of DAB-stained ChAT + neurons in the MSN region; $n=10(2 \mathrm{~N}), \mathrm{n}=11(2 \mathrm{~N}-\mathrm{NFMD}), \mathrm{n}=14(\mathrm{Ts} 2)$ and $n=11$ (Ts2-NFMD). g-h. Abnormal morphology of ChAT+ neurons was seen in the MSN of Ts2 mice, with swelling (yellow arrows), perikaryal atrophy (red arrows) and below normal ChAT immunoreactivity intensity (green arrows) in the representative images (scale bar,10 $\mu \mathrm{m}$ ). Statistical significance is represented by asterisks $* p \leq 0.05, * * p \leq 0.01, * * * p \leq 0.005$. 
Figure 2. Slower decline of LTP and normalized scores of behavioral tests in Ts2 mice treated with NFMD

a. Input/output relationship plots of hippocampal slices from $2 \mathrm{~N}$ and $\mathrm{Ts} 2$ mice treated with either vehicle or NFMD $(n=15-20$ slices from $n=4$ mice for the Ts 2 group and from $n=5$ mice for the other treatment groups). b. Plots of LTP in the Schaffer collateral synapses (CA3-CA1) induced by theta-burst stimulation (TBS) of the mice from four treatment groups. c. Averages of fEPSP slopes at 1, 50, and 110 min following tetanic stimulation; NFMD treatment modestly increased the fEPSP slope of Ts 2 mice $(18 \%, p<0.05)$ at the 110 min time point. d. Novel Object Recognition (NOR) test represented by recognition index in $2 \mathrm{~N}$ and $\mathrm{Ts} 2$ mice before (Pre) and after (Post) 4 weeks of NFMD treatments ( $n=6-8$ mice for $2 \mathrm{~N}$ and Ts 2 mice). e. Open Field test results including speed, distance and percentage of time spent in thigmotaxis for $2 \mathrm{~N}$ and Ts 2 mice before (Pre) and after (Post) 4 weeks of NFMD treatments are represent as bar graphs (n=610 mice for $2 \mathrm{~N}$ and Ts 2 mice). Statistical significance is represented by asterisks $* p \leq 0.05$, $* * p \leq 0.01, * * * p \leq 0.005$. 
Figure 3. The positive effects of Neflamapimod on pathology and function in Ts2 mice appear to be mediated by inhibition of the p38a kinase pathway and related to BACE1 and $\beta C T F$ reductions

a. Western blot analysis of p38 $\alpha$, phosphorylated-p38 (p-p38), and downstream substrates MK2 and MNK1 in tissue homogenates of the brain cortex after 2 weeks of either vehicle or NFMD treatment ( $\mathrm{n}=5$ for Ts2-NFMD, and $\mathrm{n}=6$ for other treatment groups). $\mathrm{b}$. Quantification of the Western blot images shown in a with Image J. c-d. Subsequent Western blot analysis for full length APP (APPfl), APP- $\beta C T F$ and BACE1 and Western blot image quantification. Statistical significance is represented by asterisks $* p \leq 0.05, * * p \leq 0.01, * * * p \leq 0.005$. 
Figure 4. Descriptive Analyses of Outcomes Related to Cholinergic Function in the Clinical Study

Descriptive mean data for observed data (i.e., all reported data) is shown for each outcome measure. Because of Covid-19 lockdowns, not all study visits could take place onsite, and for all but the CDR-SB the assessments could only occur onsite, the number of participants, therefore, varies during the course of the study; the number of participants with data at indicated study visit are shown by treatment group below each outcome measure. See Table 1 for output of statistical analyses by mixed model for repeated measures (MMRM) which includes evaluated data from each study visit. a. Cognition, as assessed by a Neuropsychological Test Battery (NTB) composed of six tests that individually assess attention, executive function or visuospatial function. Mean (s.e.m.) absolute value of the composite z-score that includes results of all six tests are shown. b. Attention, as assessed by an Attention composite z-score that includes results on the two tests within the NTB that evaluate information processing speed, Detection and Identification. Mean (s.e.m.) absolute value of the attention composite z-score is shown. c. Cognition and function, as assessed by the Clinical Dementia Rating scale sum of boxes (CDRSB). Mean (s.e.m.) changes from baseline to indicated study visit are shown. d. Motor function, as assessed by the Timed Up and Go (TUG) test. Mean (s.e.m.) changes from baseline to indicated study visit in time required in seconds to complete the TUG test are shown. 


\section{Figure 5. Improvement in change from baseline between neflamapimod treatment and placebo (MMRM analysis) by baseline plasma ptau181 status}

For each outcome measure, the difference between placebo and either neflamapimod treatment (combined 40mg BID and 40mg TID recipients; left panel for each measure) or neflamapimod 40mg TID (right panel) from the statistical analysis by mixed model for repeated measures is shown grouped by whether baseline plasma ptau181 was $<2.2 \mathrm{pg} / \mathrm{mL}$ (i.e., no AD co-pathology, "pure DLB") or $\geq 2.2 \mathrm{pg} / \mathrm{mL}$ (presence of AD co-pathology, indicating mixed AD-DLB. For all four endpoints the level of improvement relative to placebo in pure DLB appears better than that in mixed AD-DLB. a. Composite z-score of the Neuropsychological Test Battery (NTB) composed of six tests that individually assess attention, executive function or visuospatial function. b. Attention composite z-score, as assessed by an Attention composite z-score that includes results on the two tests within the NTB that evaluate information processing speed, Detection and Identification. c. Cognition and function, as assessed by the Clinical Dementia Rating scale sum of boxes (CDR-SB) total score. d. Motor function, as assessed time in seconds required to complete the Timed Up and Go (TUG) test. Statistical significance for the individual comparisons is represented by asterisks $* p \leq 0.05, * * p \leq 0.01, * * * p \leq 0.005$. 


\section{ONLINE METHODS:}

\section{Preclinical Study Design}

Mouse experimentation and animal care were approved by the Institutional Animal Care and Use Committee (IACUC) of the Nathan S. Kline Institute. Ts2 (Stock No. 004850) mice, and wild type breeding partner (B6EiC3SnF1/J, Stock No. 001875) from the same colony were obtained from The Jackson Laboratory (Bar Harbor, ME).

The test compound neflamapimod (manufacturer lot number M10140) and instructions for formulation preparation, route of administration and dosing volume were provided by EIP Pharma, Inc. as for previously described studies ${ }^{17,61}$. Both wild-type $(2 \mathrm{~N})$ and Ts2 mice (n=8-10 per group, targeting an equal number of female and male mice) were treated for the duration of two to four weeks, twice-daily by oral gavage at a $5 \mathrm{ml} / \mathrm{kg}$ dosing volume with vehicle (1\% (w/v) Pluronic F108, Sigma) or neflamapimod that was freshly dissolved in 1\% (w/v/) Pluronic F108 at a final concentration of $0.6 \mathrm{mg} / \mathrm{ml}$ to administer the test compound at $3 \mathrm{mg} / \mathrm{kg}$. Treatment was initiated at 4.7-6.4 months of age for $2 \mathrm{~N}$ (control) and Ts 2 mice, when endosomal pathology is evident and cholinergic neuronal loss is developing in Ts2 mice. To assess the health status of the animals throughout the studies and ensure their suitability for use in research, their general health status was monitored daily, and body weight was determined weekly.

One hour after either the terminal vehicle or neflamapimod treatment, mice were euthanized by displacement of air with $100 \% \mathrm{CO}_{2}$ and decapitated within 5 minutes. Whole mouse brains were collected and split into two brain hemispheres. The hippocampal sections were dissected out for electric physiology testing, while the remainders of the hemibrains were stored at $-80^{\circ} \mathrm{C}$ for further biochemistry analysis. For immunocytochemistry analysis, mice were anesthetized with a mixture of ketamine $(100 \mathrm{mg} / \mathrm{kg}$ body weight $)$ and xylazine $(10 \mathrm{mg} / \mathrm{kg}$ body 
weight) and transcardially perfused with $4 \%$ paraformaldehyde and brain tissues were sectioned into $40 \mu \mathrm{m}$ thick slices with a vibratome (Leica VT1000S).

\section{Immunocytochemistry and Western Blot Analysis of Preclinical Samples}

Immunohistochemistry was performed on vibratome sections ${ }^{12,62}$ using commercial antibodies against Rab5-GTP (NewEast, 26911; 1:50), ChAT (Millipore Sigma; AB144; 1:250), Rab5 (Abcam; 18211; 1:1000) and visualized either with biotinylated (Vector Laboratories; 1:500) or fluorescence-conjugated secondary antibodies (Fisher Sci, 1:500) as previous described $^{12,42,63,64}$. For protein analyses, mouse brain tissues were homogenized ${ }^{65}$ and western blot analyses were performed with antibodies against APP (c1/6.1; 1:1000), $\beta$ CTF (M3.2, 1:250) ${ }^{12,66}$, BACE1 (Rockland; 200-401-984; 1:500), MAPKAPK-2 (MK2; Cell Signaling; 12155; 1:500), phospho-MK2 (Cell Signaling; 3007; 1:500), p38 MAPK (p38 $\alpha$; Cell Signaling; 9218; 1:500), phosphor-p38 (Santa Cruz; 166182; 1:500), MNK1 (Cell Signaling; 2195; 1:500), pMNK1 (Cell Signaling; 2111; 1:500), $\beta$-actin (Santa Cruz Biotechnology; sc-47778; 1:2000). All the secondary antibodies for western blot analyses were used according to the manufacturer's recommendations (Jackson ImmunoResearch Laboratories, PA). A digital gel imager (Syn-gene G:Box XX9) was used to capture the ECL images and band intensities were quantified with Fiji/ImageJ (https://imagej.net/Fiji) using $\beta$-actin as an internal control. Sizes of Rab5+ endosomes were determined by Image J, as previously described ${ }^{12,42,63}$.

\section{Stereological counting of MS-ChAT+ neurons in Ts 2 and wild-type mice}

The numbers of ChAT-immunoreactive BFCNs in MS region were determined using the optical fractionator method ${ }^{67}$ using ImageJ software as previously described ${ }^{12,68}$. Cells were sampled with a grid of optical dissector sampling sites, on every third 40-micron thick coronal 
section through the medial septal area. At each sampling site a z-stack of 2-micron spaced images was collected using a 40x oil-immersion objective, and 160 x 116 micron counting box was drawn onto the z-stack, with an upper guard zone of 2 microns (1 slice), counting box of 4 microns, and lower guard zone of the remaining slices. All counts were adjusted for on-slide zaxis shrinkage.

\section{Electrophysiology of Ts2 and wild-type mice}

6-7-month-old $2 \mathrm{~N}$ and Ts2 mice, either treated with vehicle or with neflamapimod for 4 weeks ( $\mathrm{n}=4-5$ ) were used to measure long term potentiation (LTP) in CA1 hippocampal regions, as previous described ${ }^{36,42,68}$. Transverse hippocampal slices $(400 \mu \mathrm{m})$ were cut and placed in a recording chamber which was filled with artificial CSF (ACSF) at $29^{\circ} \mathrm{C}$ and maintained consistent at $95 \% \mathrm{O}_{2}$ and $5 \% \mathrm{CO}_{2}$. Field EPSPs (fEPSPs) were recorded by placing both the stimulating and the recording electrodes in hippocampal CA1 stratum radiatum, while basal synaptic transmission (BST) was determined by plotting the stimulus voltages over the slopes of fEPSPs. LTP was induced using theta-burst stimulation and responses were recorded for $2 \mathrm{~h}$ and measured as fEPSP slope expressed as percentage of baseline ${ }^{68}$.

\section{Preclinical evaluation of behavioral outcomes}

Open field and NOR test were performed according to previous publications ${ }^{42,69}$. A multiple unit open field maze with four activity chambers was used and activity of the mouse was recorded with a digital video camera, which was linked to a computer. For NOR, 10 mins of exploration time was used for the analysis with CowLog 3 , an opensource software ${ }^{70}$. The results were presented as Recognition Index (RI), which was defined as time exploring the novel object divided by the sum of time exploring both the novel and familiar objects as previous defined ${ }^{42}$. 
For open field, 6 mins exploration time was used for the analysis with ToxTrac ${ }^{71}$, and the following parameters including speed, travel distance and percentage of time spend in thigmotaxis (all edges of the chamber) was determined for each mice.

\section{Statistical analysis of preclinical study}

All quantitative data were subjected to two-tailed unpaired Student's $t$-test for single comparison, and one-way ANOVA analysis for multiple comparisons with post-hoc Tukey's analysis using GraphPad Prism 8. Most data are represented as box and whisker plots, also showing group means as crosses (see Figures). For data involving fewer than 6 repeats, mean \pm SEM were represented in bar graphs with individual data points. Statistical significance is represented by asterisks $* p \leq 0.05, * * p \leq 0.01, * * * p \leq 0.005$. No statistical methods were used to predetermine sample sizes, but our sample sizes are chosen based on similar data in previous publications s, $12,17,36,42,68,69$.

\section{Clinical Study Design}

Study EIP19-NFD-501 was a Phase 2, multicenter, randomized, double-blind, placebocontrolled, proof-of-principle study of neflamapimod $40 \mathrm{mg}$ or matching placebo conducted at 24 centers, 22 in the US and 2 in the Netherlands (see Acknowledgements for list of investigators). The first participant was enrolled on 30 September 2019 and the last participant visit occurred on 14 July 2020. The study was conducted in accordance with Good Clinical Practice guidelines (reference) and the Declaration of Helsinki (reference). Applicable local/central ethics committee or IRB approvals were obtained, and all participants provided written informed consent. IRB/Ethics approvals provided by Copernicus Group IRB (CGIRB, Cary, NC), Western Institutional Review Board (WIRB, Puyallup, WA), Mayo Clinic Institutional Review Board (Rochester MN), Columbia University Medical Center Institutional 
Review Board (New York, NY), Cleveland Clinic IRB (Cleveland, OH), and Foundation Beoordeling Ethiek Biomedisch Onderzoek (BEBO, Assen, the Netherlands). The trial was registered at clinicaltrials.gov as NCT04001517 and in the EU Clinical Trials Register with EudraCT Number of 2019-001566-15.

Participants included men and women aged $\geq 55$ years with probable DLB and identified cognitive deficits, according to current consensus criteria, specifically one core clinical feature and a positive DaTscan; an MMSE score of 15-28, inclusive; and who were receiving cholinesterase inhibitor therapy (having received such therapy for greater than 3 months and on a stable dose for at least 6 weeks at the time of randomization) during Screening were eligible. If the subject had a negative DaTscan, but had historical PSG-verified RBD, the subject would have qualified.

Following completion of informed consent procedures, participants entered the Screening phase of the study. One to two Screening visits were planned, during which safety screening measures were undertaken, practice cognitive tests were performed, and the required diagnosis and cognitive impairment was confirmed. All screening assessments were to be conducted within 21 days of Day 1 (first dose of study drug), the extension to 35 days was allowed if a DaTscan ${ }^{\mathrm{TM}}$ was required to determine study eligibility. After eligibility was confirmed and before the first dose of study drug, participants were randomly assigned on a 1:1 basis by an external vendor (Suvoboda Inc, Conshocken, PA, USA) with an Interactive Response Technology (IRT) system to receive $40 \mathrm{mg}$ neflamapimod capsules or matching placebo capsules orally, BID or TID with food for 16 weeks. Capsules were supplied in blister cards, which each blister card containing 16 capsules (i.e., 1-week supply of study, with two extra capsules) for BID and 24 capsules for TID (i.e., 1-week supply of study, with three extra capsules). The blister cards were kitted, with 2 
blister cards per kit. Each kit was labeled as Study Drug and a unique identifier number, which utilized by the IRT system to assign kits to individual participants at randomization and on-study when resupply was required. Participants s followed the BID regimen if weighing $<80 \mathrm{~kg}$ or the TID regimen if weighing $\geq 80 \mathrm{~kg}$. Doses were taken within 30 minutes following a meal or snack (i.e., morning, mid-day (TID only), and evening meals) at least 3 hours apart and at approximately the same times each day throughout the study.

Dosing started on Day 1, following completion of all Baseline procedures. During the treatment period, subjects attended study center visits on Days 14, 28, 56, 84, and 112. Due to the COVID-19 global pandemic, some of these visits were permitted to be held remotely due to local restrictions to travel and in-clinic visits.

\section{Plasma ptau181 measurement}

Plasma p-Tau181 was measured in the clinical chemistry laboratory at the VU Medical Center according to the kit instructions (p-Tau181 V2 kit, Quanterix, Billerica, USA). In-house quality control plasma samples were run in duplicates at the start and the end of each plate to determine the within-run and between-run variations.

\section{Clinical Study Outcome Measures}

The primary objective was to evaluate the effect of neflamapimod on cognitive function as assessed in a study-specific Neuropsychological Test Battery (NTB) comprised of four computerized tests from the Cogstate ${ }^{\circledR}$ cognitive testing battery (Detection, Identification, One Card Learning, One Back) and two verbal fluency tests (Letter Fluency Test, Category Fluency Test) that were recorded on paper. 
Secondary objectives included the (1) evaluate effects on cognition and function, as assessed by the Clinical Dementia Rating Scale sum-of-boxes (CDR-SB), (2) assess effects on motor function, as assessed by the Timed Up and Go test, (3) Assess the effects of neflamapimod on episodic memory, as assessed by the International Shopping List Test (ISLT), (4) Assess the effects of neflamapimod on select domains of the 10-item Neuropsychiatric Inventory (NPI-10), including depression (dysphoria), anxiety, hallucinations, and agitation/aggression. At study start, assessing effects on Mini Mental Status Examination (MMSE) and EEG were additional endpoints but results of analyses of these endpoints are not reported due to the impact of Covid19-related restrictions the collection of these measures. For the MMSE, all baseline data was collected onsite. However, on-study data was collected both remotely and onsite, but inconsistently, and initial analysis demonstrated a significant effect of remote visit-based assessment that made the on-treatment data not interpretable. For the EEG, only approximately one-third of patients could have a baseline and week16 EEG recording.

\section{Clinical Study Statistical Analysis}

Except for the cognitive tests within the Cogstate Battery (four within NTB, and the ISLT) all data was captured by Worldwide Clinical Trials via an electronic data capture (EDC) and after all queries were resolved and database was locked, SAS datasets were provided to Anoixis corporation for statistical analysis. The data from the Cogstate tests were processed at Cogstate and provided directly to Anoixis.

Due to the various scales among different tests in the NTB and the need to have equal weights for deriving composite score metrics performance on each, the tests were standardized relative to baseline data from all randomized subjects (i.e., the individual test core was converted 
to a z-score by subtracting the study sample's mean at baseline from the score and dividing by the standard deviation (SD) of the study sample's baseline). The NTB composite z-score was calculated as the average of the z-scores from the component of the six individual tests in the composite. For the purposes of facilitating further understanding of the drug effects on the cholinergic system, an exploratory Attention composite endpoint made up of the two tests in NTB that evaluate information processing speed, the identification and detection tests, was calculated and evaluated.

As pre-specified in the protocol, the analyses of all efficacy endpoints (primary, secondary and exploratory) used a Mixed Model for Repeated Measures (MMRM) analysis method with change from baseline as the dependent variable, with a fixed effect on treatment extended to baseline composite score and study visit as covariates. There was a fixed effect on treatment that was extended to baseline composite score and study visit. The interaction term (i.e., scheduled visit by treatment) was considered. The random effect was modeled at subject level first and was extended to other covariates if there were no numerical integration issues. 


\section{References}

1. Schmitz, T.W., Spreng, R.N. \& The-Alzheimer's-Disease-Neuroimaging-Initiative. Basal forebrain degeneration precedes and predicts the cortical spread of Alzheimer's pathology. Nat Commun 7, 13249 (2016).

2. Hampel, H., et al. Revisiting the Cholinergic Hypothesis in Alzheimer's Disease: Emerging Evidence from Translational and Clinical Research. J Prev Alzheimers Dis 6, 2-15 (2019).

3. Fernandez-Cabello, S., et al. Basal forebrain volume reliably predicts the cortical spread of Alzheimer's degeneration. Brain 143, 993-1009 (2020).

4. Wilson, J., et al. Cholinergic Basal Forebrain Volumes Predict Gait Decline in Parkinson's Disease. Mov Disord 36, 611-621 (2021).

5. Jarzebowski, P., Tang, C.S., Paulsen, O. \& Hay, Y.A. Impaired spatial learning and suppression of sharp wave ripples by cholinergic activation at the goal location. Elife 10(2021).

6. Nixon, R.A. Amyloid precursor protein and endosomal-lysosomal dysfunction in Alzheimer's disease: inseparable partners in a multifactorial disease. FASEB J 31, 27292743 (2017).

7. Kim, S., et al. Evidence that the rab5 effector APPL1 mediates APP-betaCTF-induced dysfunction of endosomes in Down syndrome and Alzheimer's disease. Mol Psychiatry 21, 707-716 (2016).

8. Salehi, A.H., et al. NRAGE, a novel MAGE protein, interacts with the p75 neurotrophin receptor and facilitates nerve growth factor-dependent apoptosis. Neuron 27, 279-288 (2000). 
9. Cataldo, A.M., et al. Endocytic pathway abnormalities precede amyloid beta deposition in sporadic Alzheimer's disease and Down syndrome: differential effects of APOE genotype and presenilin mutations. Am J Pathol 157, 277-286 (2000).

10. Cataldo, A.M., et al. Down syndrome fibroblast model of Alzheimer-related endosome pathology: accelerated endocytosis promotes late endocytic defects. Am J Pathol 173, 370$384(2008)$.

11. Holtzman, D.M., et al. Developmental abnormalities and age-related neurodegeneration in a mouse model of Down syndrome. Proc Natl Acad Sci U S A 93, 13333-13338 (1996).

12. Jiang, Y., et al. Partial BACE1 reduction in a Down syndrome mouse model blocks Alzheimer-related endosomal anomalies and cholinergic neurodegeneration: role of APPCTF. Neurobiol Aging 39, 90-98 (2016).

13. Cooper, J.D., et al. Failed retrograde transport of NGF in a mouse model of Down's syndrome: reversal of cholinergic neurodegenerative phenotypes following NGF infusion. Proc Natl Acad Sci U S A 98, 10439-10444 (2001).

14. Chen, X.Q. \& Mobley, W.C. Exploring the Pathogenesis of Alzheimer Disease in Basal Forebrain Cholinergic Neurons: Converging Insights From Alternative Hypotheses. Front Neurosci 13, 446 (2019).

15. Cavalli, V., et al. The stress-induced MAP kinase p38 regulates endocytic trafficking via the GDI:Rab5 complex. Mol Cell 7, 421-432 (2001).

16. Gibbs, K.L., et al. Inhibiting p38 MAPK alpha rescues axonal retrograde transport defects in a mouse model of ALS. Cell Death Dis 9, 596 (2018). 
17. Alam, J.J. Selective brain-targeted antagonism of p38 MAPKalpha reduces hippocampal IL-1beta levels and improves Morris water maze performance in aged rats. $J$ Alzheimers Dis 48, 219-227 (2015).

18. Alam, J., Blackburn, K. \& Patrick, D. Neflamapimod: Clinical phase 2b-ready oral small molecule inhibitor of p38alpha to reverse synaptic dysfunction in early Alzheimer's disease. J. Prevention of Alzheimer's Disease 4, 273-278 (2017).

19. Prins, N.D., et al. A phase 2 double-blind placebo-controlled 24-week treatment clinical study of the p38 alpha kinase inhibitor neflamapimod in mild Alzheimer's disease. Alzheimer's Research \& Therapy 13, 106 (2021).

20. Grothe, M.J., et al. Atrophy of the cholinergic basal forebrain in dementia with Lewy bodies and Alzheimer's disease dementia. J Neurol 261, 1939-1948 (2014).

21. Perry, E.K. Nerve growth factor and the basal forebrain cholinergic system: a link in the etiopathology of neurodegenerative dementias? Alzheimer Dis Assoc Disord 4, 1-13 (1990).

22. Duda, J.E. Pathology and neurotransmitter abnormalities of dementia with Lewy bodies. Dement Geriatr Cogn Disord 17 Suppl 1, 3-14 (2004).

23. White, A., Pargellis, C.A., Studts, J.M., Werneburg, B.G. \& Farmer, B.T., 2nd. Molecular basis of MAPK-activated protein kinase 2:p38 assembly. Proc Natl Acad Sci U S A 104, 6353-6358 (2007).

24. Trempolec, N., Dave-Coll, N. \& Nebreda, A.R. SnapShot: p38 MAPK substrates. Cell 152, 924-924 e921 (2013). 
25. Lawson, S.K., Dobrikova, E.Y., Shveygert, M. \& Gromeier, M. p38alpha mitogenactivated protein kinase depletion and repression of signal transduction to translation machinery by miR-124 and -128 in neurons. Mol Cell Biol 33, 127-135 (2013).

26. Schnoder, L., et al. Deficiency of Neuronal p38alpha MAPK Attenuates Amyloid Pathology in Alzheimer Disease Mouse and Cell Models through Facilitating Lysosomal Degradation of BACE1. J Biol Chem 291, 2067-2079 (2016).

27. McKeith, I.G., et al. Diagnosis and management of dementia with Lewy bodies: Fourth consensus report of the DLB Consortium. Neurology 89, 88-100 (2017).

28. Hall, S., et al. Plasma Phospho-Tau Identifies Alzheimer's Co-Pathology in Patients with Lewy Body Disease. Mov Disord 36, 767-771 (2021).

29. McKeith, I.G., Wesnes, K.A., Perry, E. \& Ferrara, R. Hallucinations predict attentional improvements with rivastigmine in dementia with lewy bodies. Dement Geriatr Cogn Disord 18, 94-100 (2004).

30. Niewiadomska, G., Komorowski, S. \& Baksalerska-Pazera, M. Amelioration of cholinergic neurons dysfunction in aged rats depends on the continuous supply of NGF. Neurobiol Aging 23, 601-613 (2002).

31. Loy, B., et al. p38alpha and p38beta mitogen-activated protein kinases determine cholinergic transdifferentiation of sympathetic neurons. J Neurosci 31, 12059-12067 (2011).

32. Scali, C., et al. The selective cyclooxygenase-2 inhibitor rofecoxib suppresses brain inflammation and protects cholinergic neurons from excitotoxic degeneration in vivo. Neuroscience 117, 909-919 (2003). 
33. Miyakawa, T., Yamada, M., Duttaroy, A. \& Wess, J. Hyperactivity and intact hippocampus-dependent learning in mice lacking the M1 muscarinic acetylcholine receptor. J Neurosci 21, 5239-5250 (2001).

34. Valuskova, P., Riljak, V., Forczek, S.T., Farar, V. \& Myslivecek, J. Variability in the Drug Response of M4 Muscarinic Receptor Knockout Mice During Day and Night Time. Front Pharmacol 10, 237 (2019).

35. Pepeu, G., Scali, C. \& Giovannini, M.G. Chapter 25. The role of cholinergic system in novel object recognition. in Handbook of Behavioral Neuroscience (eds. Ennaceur, A. \& deSouza Silva, M.) 371-378 (Elsevier, Amsterdam, 2018).

36. Kaur, G., et al. Glutamatergic transmission aberration: a major cause of behavioral deficits in a murine model of Down's syndrome. J Neurosci 34, 5099-5106 (2014).

37. Kurt, M.A., Kafa, M.I., Dierssen, M. \& Davies, D.C. Deficits of neuronal density in CA1 and synaptic density in the dentate gyrus, CA3 and CA1, in a mouse model of Down syndrome. Brain Res 1022, 101-109 (2004).

38. Lorenzi, H.A. \& Reeves, R.H. Hippocampal hypocellularity in the Ts65Dn mouse originates early in development. Brain Res 1104, 153-159 (2006).

39. Dickinson-Anson, H., et al. Acetylcholine-secreting cells improve age-induced memory deficits. Mol Ther 8, 51-61 (2003).

40. Conner, J.M., Chiba, A.A. \& Tuszynski, M.H. The basal forebrain cholinergic system is essential for cortical plasticity and functional recovery following brain injury. Neuron $\mathbf{4 6}$, 173-179 (2005).

41. Mirza Agha, B., et al. Cholinergic upregulation by optogenetic stimulation of nucleus basalis after photothrombotic stroke in forelimb somatosensory cortex improves endpoint 
and motor but not sensory control of skilled reaching in mice. J Cereb Blood Flow Metab 41, 1608-1622 (2021).

42. Pensalfini, A., et al. Endosomal Dysfunction Induced by Directly Overactivating Rab5 Recapitulates Prodromal and Neurodegenerative Features of Alzheimer's Disease. Cell Rep 33, 108420 (2020).

43. Ge, B., et al. MAPKK-independent activation of p38alpha mediated by TAB1-dependent autophosphorylation of p38alpha. Science 295, 1291-1294 (2002).

44. Burton, J.C., Antoniades, W., Okalova, J., Roos, M.M. \& Grimsey, N.J. Atypical p38 Signaling, Activation, and Implications for Disease. Int J Mol Sci 22(2021).

45. Canovas, B. \& Nebreda, A.R. Diversity and versatility of p38 kinase signalling in health and disease. Nat Rev Mol Cell Biol 22, 346-366 (2021).

46. Alam, J. \& Scheper, W. Targeting neuronal MAPK14/p38alpha activity to modulate autophagy in the Alzheimer disease brain. Autophagy 12, 2516-2520 (2016).

47. Colie, S., et al. Neuronal p38alpha mediates synaptic and cognitive dysfunction in an Alzheimer's mouse model by controlling beta-amyloid production. Sci Rep 7, 45306 (2017).

48. Schnoder, L., et al. P38alpha-MAPK phosphorylates Snapin and reduces Snapin-mediated BACE1 transportation in APP-transgenic mice. FASEB J 35, e21691 (2021).

49. Mamikonyan, E., Xie, S.X., Melvin, E. \& Weintraub, D. Rivastigmine for mild cognitive impairment in Parkinson disease: a placebo-controlled study. Mov Disord 30, 912-918 (2015).

50. Matsunaga, S., Kishi, T., Yasue, I. \& Iwata, N. Cholinesterase Inhibitors for Lewy Body Disorders: A Meta-Analysis. Int J Neuropsychopharmacol 19(2015). 
51. Nazmuddin, M., Philippens, I. \& van Laar, T. Electrical stimulation of the nucleus basalis of meynert: a systematic review of preclinical and clinical data. Sci Rep 11, 11751 (2021).

52. Wilkins, K.B., Parker, J.E. \& Bronte-Stewart, H.M. Gait variability is linked to the atrophy of the Nucleus Basalis of Meynert and is resistant to STN DBS in Parkinson's disease. Neurobiol Dis 146, 105134 (2020).

53. Dalrymple, W.A., et al. Cholinergic nucleus 4 atrophy and gait impairment in Parkinson's disease. J Neurol 268, 95-101 (2021).

54. Rockwood, K., Black, S.E., Robillard, A. \& Lussier, I. Potential treatment effects of donepezil not detected in Alzheimer's disease clinical trials: a physician survey. Int $J$ Geriatr Psychiatry 19, 954-960 (2004).

55. Dumas, J.A. \& Newhouse, P.A. The cholinergic hypothesis of cognitive aging revisited again: cholinergic functional compensation. Pharmacol Biochem Behav 99, 254-261 (2011).

56. Bracco, L., Bessi, V., Padiglioni, S., Marini, S. \& Pepeu, G. Do cholinesterase inhibitors act primarily on attention deficit? A naturalistic study in Alzheimer's disease patients. $J$ Alzheimers Dis 40, 737-742 (2014).

57. Teipel, S.J., et al. Predictors of cognitive decline and treatment response in a clinical trial on suspected prodromal Alzheimer's disease. Neuropharmacology 108, 128-135 (2016).

58. Edmonds, E.C., et al. Unmasking the benefits of donepezil via psychometrically precise identification of mild cognitive impairment: A secondary analysis of the ADCS vitamin E and donepezil in MCI study. Alzheimers Dement (N Y) 4, 11-18 (2018).

59. Dubois, B., et al. Donepezil decreases annual rate of hippocampal atrophy in suspected prodromal Alzheimer's disease. Alzheimers Dement 11, 1041-1049 (2015). 
60. Cavedo, E., et al. Reduced basal forebrain atrophy progression in a randomized Donepezil trial in prodromal Alzheimer's disease. Sci Rep 7, 11706 (2017).

61. Alam, J.J., Krakovsky, M., Germann, U. \& Levy, A. Continuous administration of a p38alpha inhibitor during the subacute phase after transient ischemia-induced stroke in the rat promotes dose-dependent functional recovery accompanied by increase in brain BDNF protein level. PLoS One 15, e0233073 (2020).

62. Cataldo, A.M., et al. App gene dosage modulates endosomal abnormalities of Alzheimer's disease in a segmental trisomy 16 mouse model of down syndrome. J Neurosci $\mathbf{2 3}, 6788$ 6792 (2003).

63. Jiang, Y., et al. Alzheimer's-related endosome dysfunction in Down syndrome is Abetaindependent but requires APP and is reversed by BACE-1 inhibition. Proc Natl Acad Sci US A 107, 1630-1635 (2010).

64. Smiley, J.F., et al. Selective reduction of cerebral cortex GABA neurons in a late gestation model of fetal alcohol spectrum disorder. Alcohol 49, 571-580 (2015).

65. Schmidt, S.D., Jiang, Y., Nixon, R.A. \& Mathews, P.M. Tissue processing prior to protein analysis and amyloid-beta quantitation. Methods Mol Biol 299, 267-278 (2005).

66. Choi, J.H., et al. Age-dependent dysregulation of brain amyloid precursor protein in the Ts65Dn Down syndrome mouse model. J Neurochem 110, 1818-1827 (2009).

67. West, M.J., Slomianka, L. \& Gundersen, H.J. Unbiased stereological estimation of the total number of neurons in thesubdivisions of the rat hippocampus using the optical fractionator. Anat Rec 231, 482-497 (1991). 
68. Basavarajappa, B.S. \& Subbanna, S. CB1 receptor-mediated signaling underlies the hippocampal synaptic, learning, and memory deficits following treatment with JWH-081, a new component of spice/K2 preparations. Hippocampus 24, 178-188 (2014).

69. Seibenhener, M.L. \& Wooten, M.C. Use of the Open Field Maze to measure locomotor and anxiety-like behavior in mice. J Vis Exp, e52434 (2015).

70. Hanninen, L. \& Pastell, M. CowLog: open-source software for coding behaviors from digital video. Behav Res Methods 41, 472-476 (2009).

71. Rodriguez, A., et al. ToxTrac: A fast and robust software for tracking organisms. Methods in Ecology and Evolution 9, 460-464 (2018). 


\section{Data Availability Statement}

Pre-clinical and clinical datasets may be obtained through contacting Prof. Ralph Nixon and Dr.

John Alam, respectively. In order to allow sufficient time for secondary publications, the clinical datasets will be made available starting September 2022, which will be two years from when database lock occurred.

\section{Acknowledgements}

AscenD-LB Clinical Study investigators: USA: K. Amadeo (U. of Rochester, Rochester, NY), G. Baras (Elite Clinical Research, Miami, FL), K. Bell (Columbia U. Medical Center, New York, NY), C. Bernick (U. of Washington, Seattle, WA), B. Boeve (Mayo Clinic, Rochester, NY), N. Bohnen (Michigan Medicine, Ann Arbor, MI), S. Gomperts (Massachusetts General Hospital, Charlestown, MA), S. Holden (U. of Colorado, Aurora, CO), D. Kaufer (U. of North Carolina, Chapel Hill, NY), S. Kesari (Pacific Neuroscience Institute, Santa Monica, CA), A. Khan (Northwest Clinical Research Center, Bellevue, WA), I. Litvan (UC San Diego Health, San Diego, CA), S. Losk (Summit Research Network, Portland, OR), R. Pahwa (U. of Kansas Medical Center, Kansas City, KS), S. Pugh (Inland Northwest Research, Spokane, WA), J. Quinn (Oregon Health and Science University, Portland OR), A. Ritter (Cleveland Clinic, Las Vegas, NV), B. Shah (UVA Health, Charlottesville, VA), D. Scharre (Ohio State Neurological Institute, Columbus, OH), M. Serruya (Jefferson U. Hospitals, Philadelphia, PA), B. Tousi (Cleveland Clinic, Cleveland, OH); Netherlands: P. Dautzenberg (Brain Research Center (BRC)

- Den Bosch, Den Bosch), A.W. Lemstra (Brain Research Center (BRC) - Amsterdam, Amsterdam). 
The preclinical study was supported by NIH grants P01AG017617 and R01AG062376 grants to R.A.N. B.S.B. and S.S. are supported by NIH grant R01 AA019443. The clinical study was funded by EIP Pharma, Inc.

The authors are very grateful to all patients and caregivers who participated in this study and to the many staff members at the clinical sites for their dedication and commitment to the clinical study. We also acknowledge the study project teams at EIP Pharma and Worldwide Clinical Trials, as well Dr. Susan Doctrow for editorial and technical assistance in preparing the manuscript.

\section{Competing Interests}

JJA, AG, KB are employees of EIP Pharma, the sponsor of the clinical study. JJA is also founder of and has stock ownership in EIP Pharma. SNG has served on Advisory Boards of Jannsen, Acadia, and Sanofi, has received consulting fees from EIP Pharma, and has received funding from the NIH, the DOD CDMRP, the Michael J. Fox Foundation, the FFFPRI, and the Lewy Body Dementia Association. NDP is CEO and co-owner of Brain Research Center. JEH reports receipt of personal fees in the past 2 years from Actinogen, AlzeCure, Aptinyx, Astra Zeneca, Athira Therapeutics, Axon Neuroscience, Axovant, Bial Biotech, Biogen Idec, BlackThornRx, Boehringer Ingelheim, Brands2life, Cerecin, Cognito, Cognition Therapeutics, Compass Pathways, Corlieve, Curasen, EIP Pharma, Eisai, G4X Discovery, GfHEU, Heptares, Ki Elements, Lundbeck, Lysosome Therapeutics, MyCognition, Neurocentria, Neurocog, Neurodyn Inc, Neurotrack, the NHS, Novartis, Novo Nordisk, Nutricia, Probiodrug, Prothena, Recognify, Regeneron, reMYND, Rodin Therapeutics, Samumed, Sanofi, Signant, Syndesi

Therapeutics, Takeda, Vivoryon Therapeutics and Winterlight Labs. Additionally, he holds stock 
options in Neurotrack Inc. and is a joint holder of patents with My Cognition Ltd. P.S. has received consultancy fees (paid to the institution) from AC Immune, Brainstorm Cell, EIP Pharma, ImmunoBrain Checkpoint, Genentech, Novartis, Novo Nordisk. He is PI of studies with AC Immune, FUJI-film/Toyama, UCB, and Vivoryon; and he is a part-time employee of Life Sciences Partners Amsterdam. 
Figure 1

a

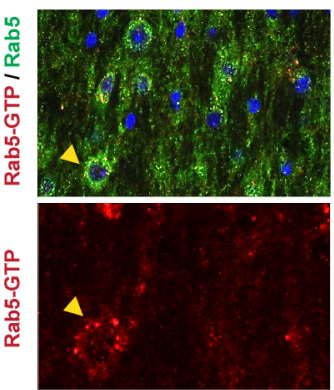

$2 \mathrm{~N}$

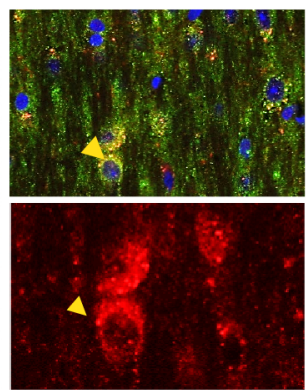

Ts2

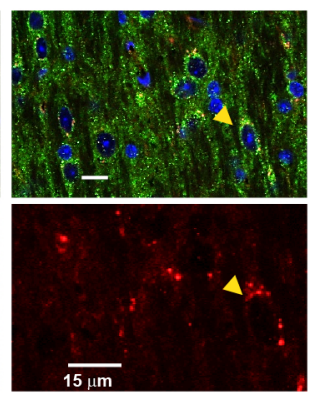

Ts2-NFMD

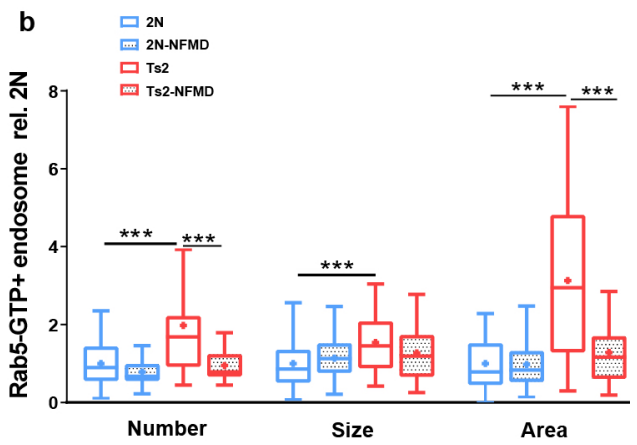

c

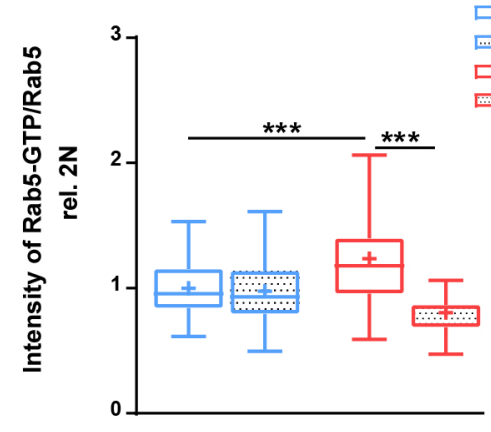

口 2N
回 2N-NFMD

$\square$ Ts2
Ts2-NFMD
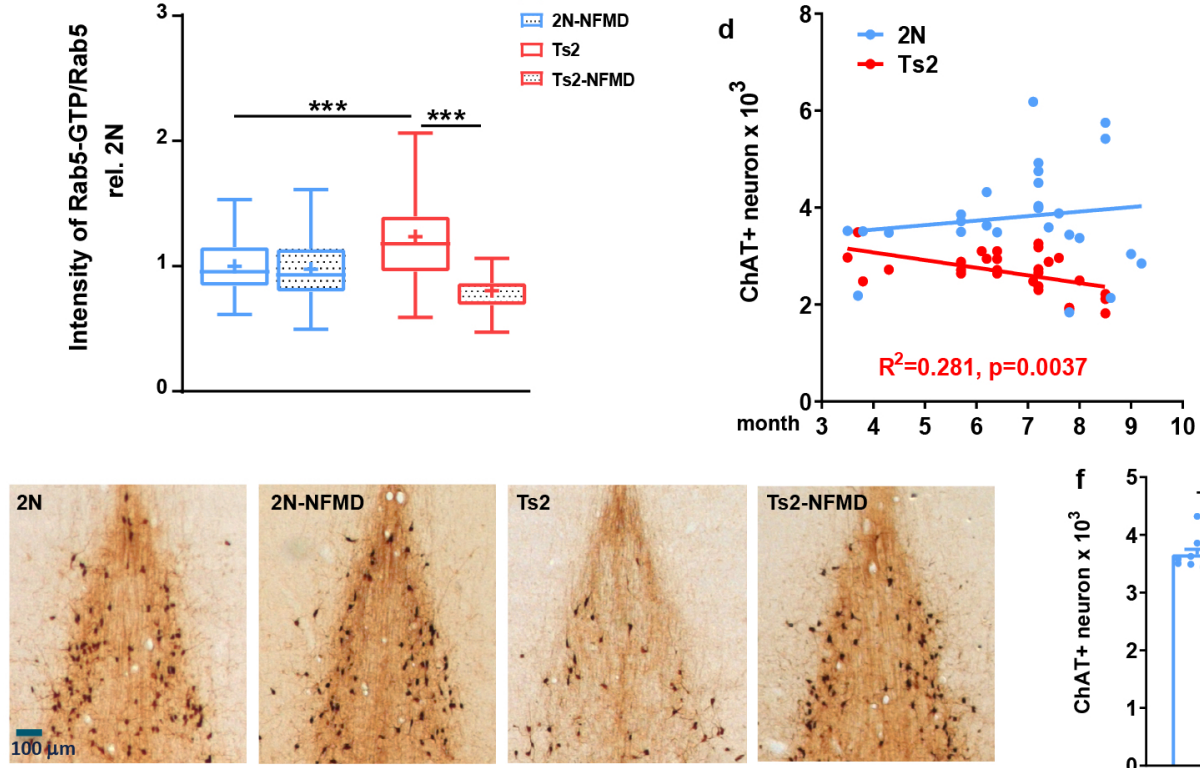

Ts2
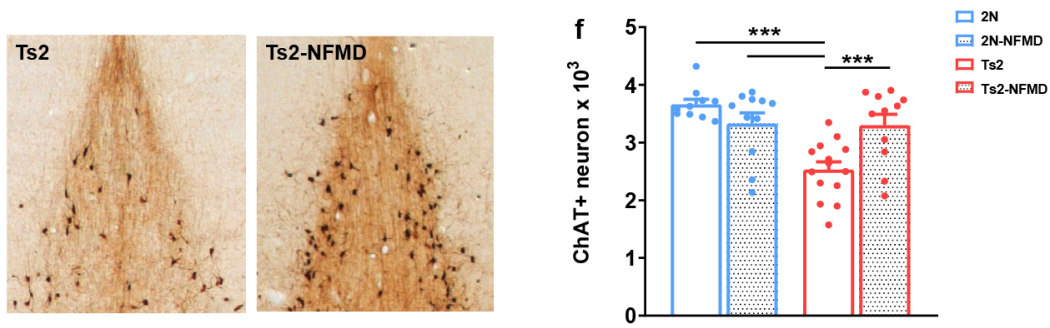

g

$2 N$

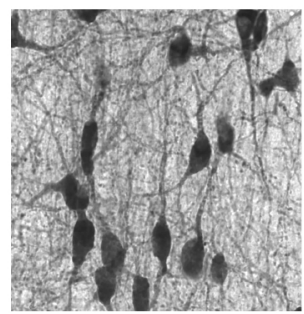

Ts2

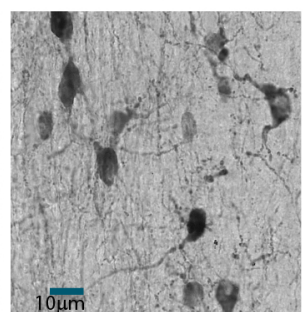

Ts2-NFMD

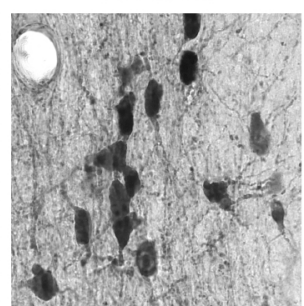

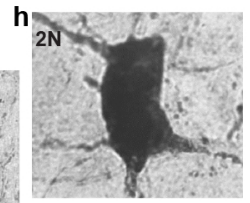

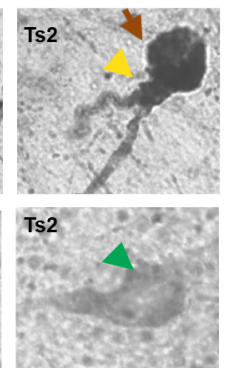

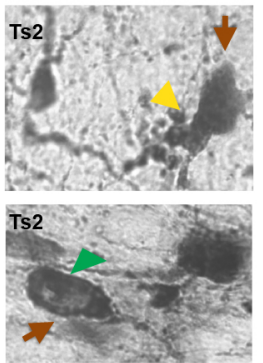


Figure 2
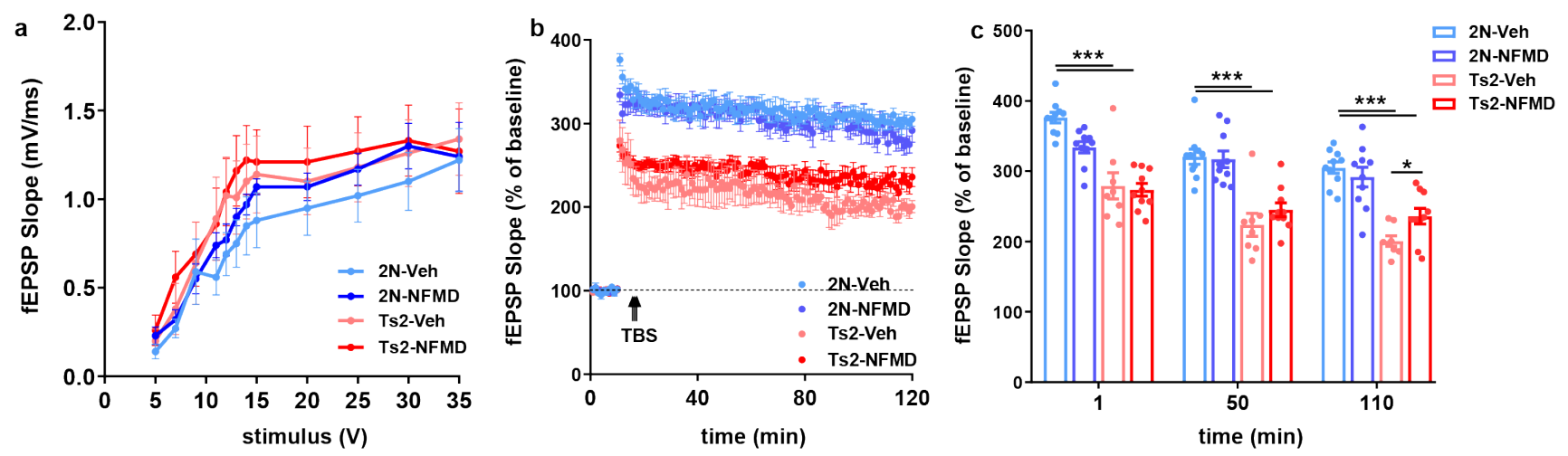

d

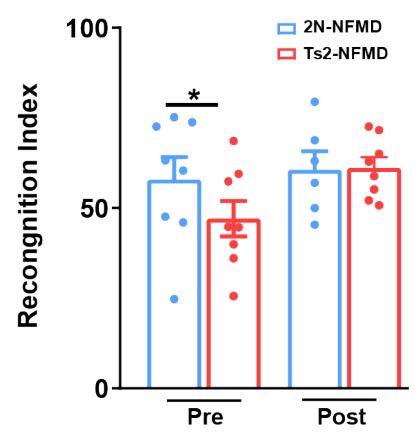

e

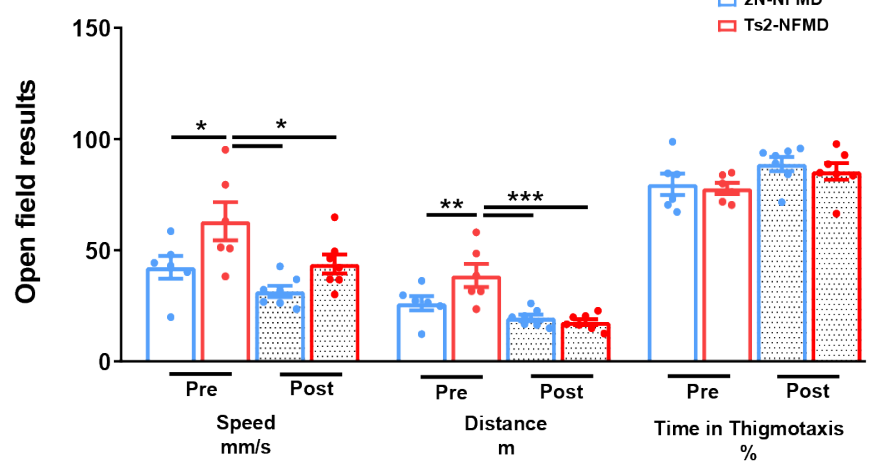


Figure 3

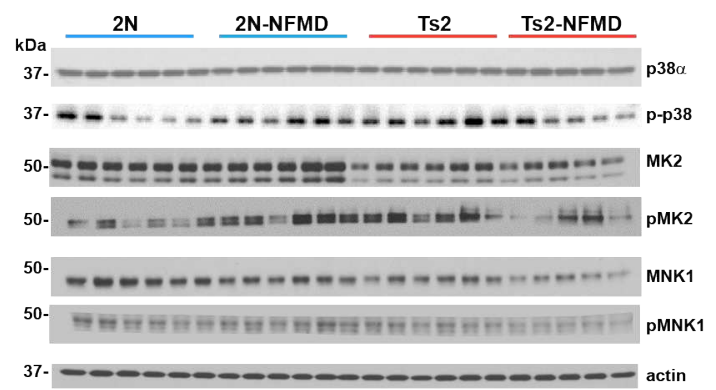

c

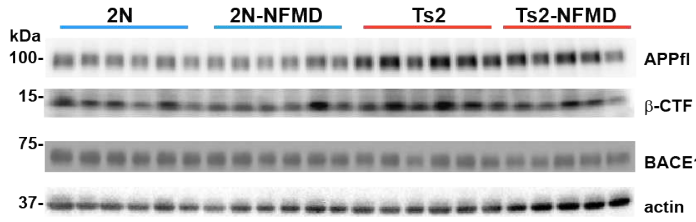

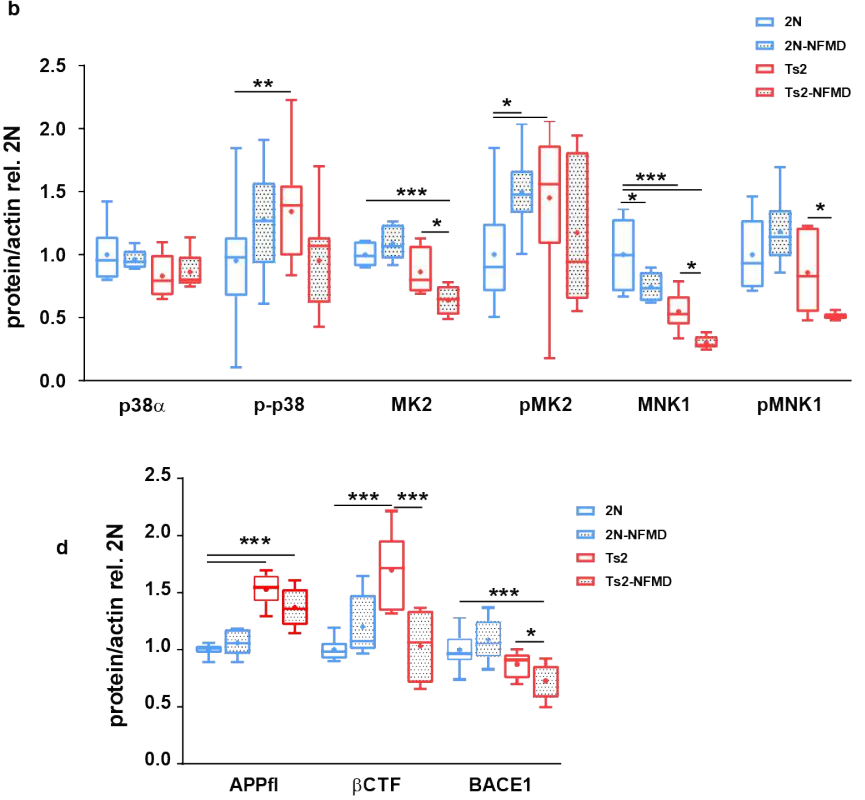


Figure 4. Descriptive (Mean change from baseline) analyses of observed data in clinical study

a. Neuropsychological Test Battery composite z-score

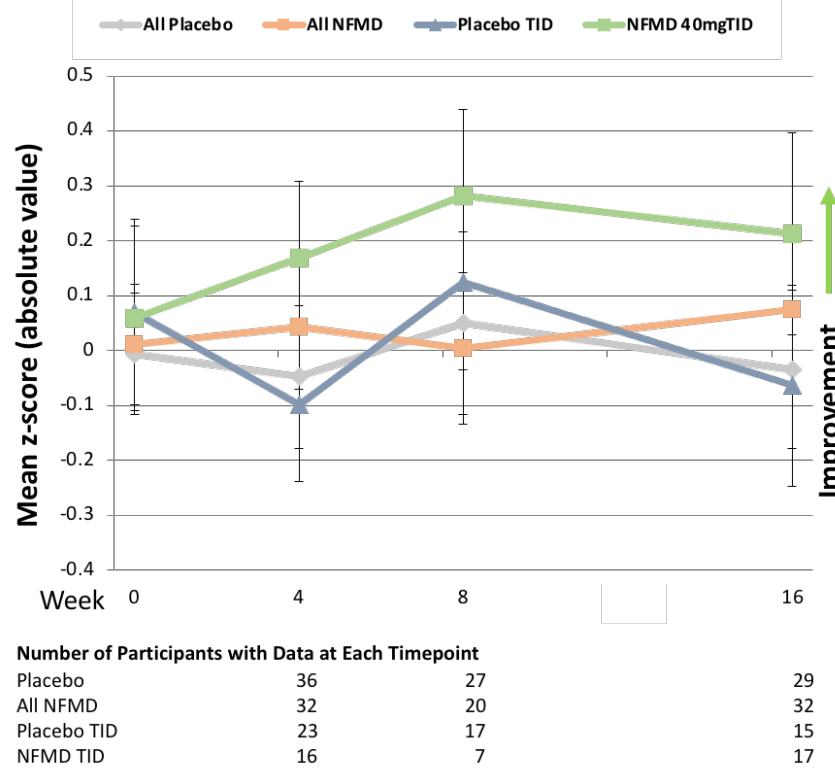

C. Clinical Dementia Rating Sum of Boxes (CDR-SB)

All Placebo $\quad$ All NFMD $\square$ Placebo TID NFMD $40 \mathrm{mg}$ TID

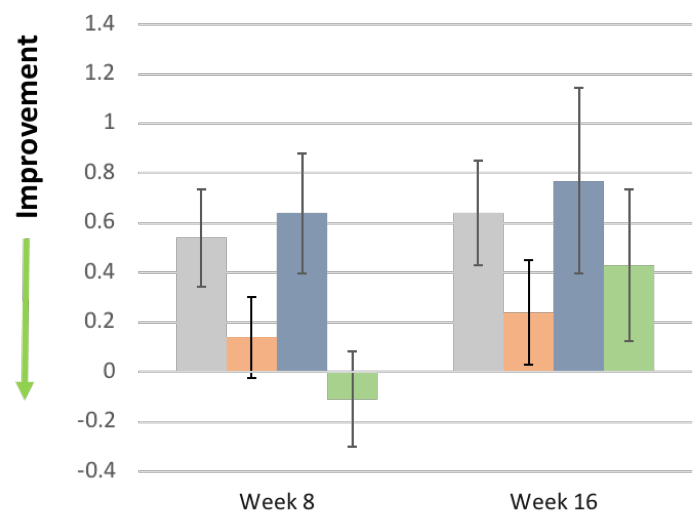

Number of Participants with Data at each Timepoint

Placebo

All NFMD

Placebo TID

NFMD TID

$\begin{array}{cr}40 & 41 \\ 37 & 40 \\ 25 & 26 \\ 18 & 20\end{array}$

b. Attention composite z-score

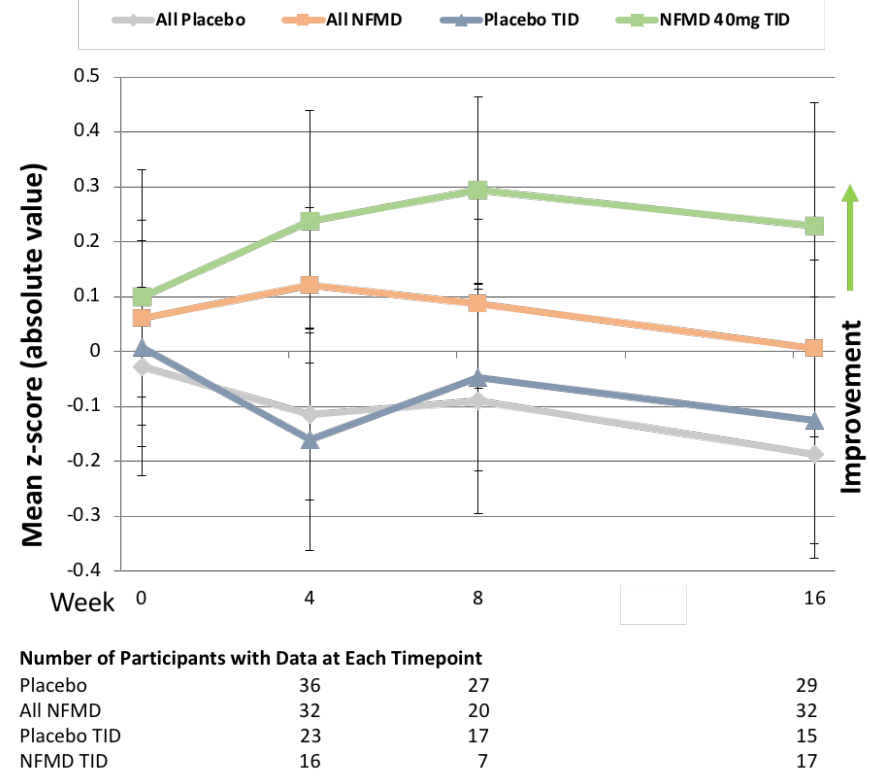

d. Time Up and Go test

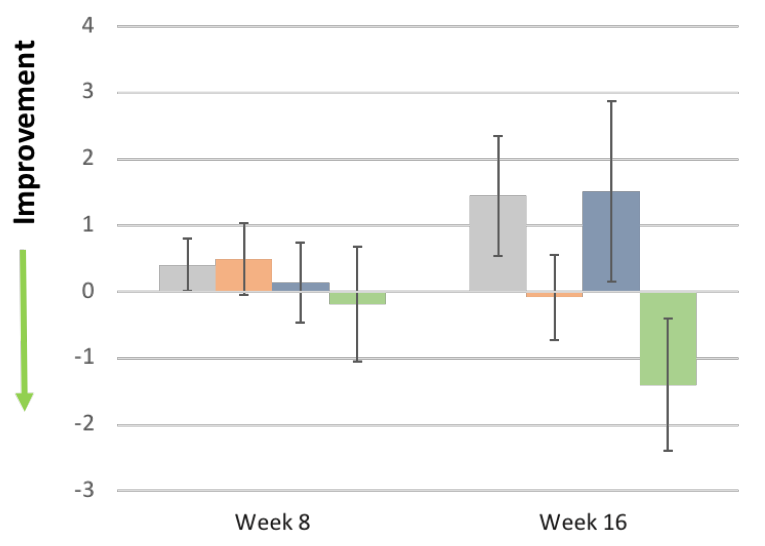

Number of Participants with Data at each Timepoint

Placebo 27

All NFMD

Placebo TID

NFMD TID

$\begin{array}{ll}27 & 31 \\ 23 & 33 \\ 16 & 19 \\ 10 & 17\end{array}$


Figure 5. Improvement in change from baseline between neflamapimod treatment and placebo (MMRM analysis) by baseline plasma ptau181 status

a. Neuropsychological Test Battery (NTB) z-score
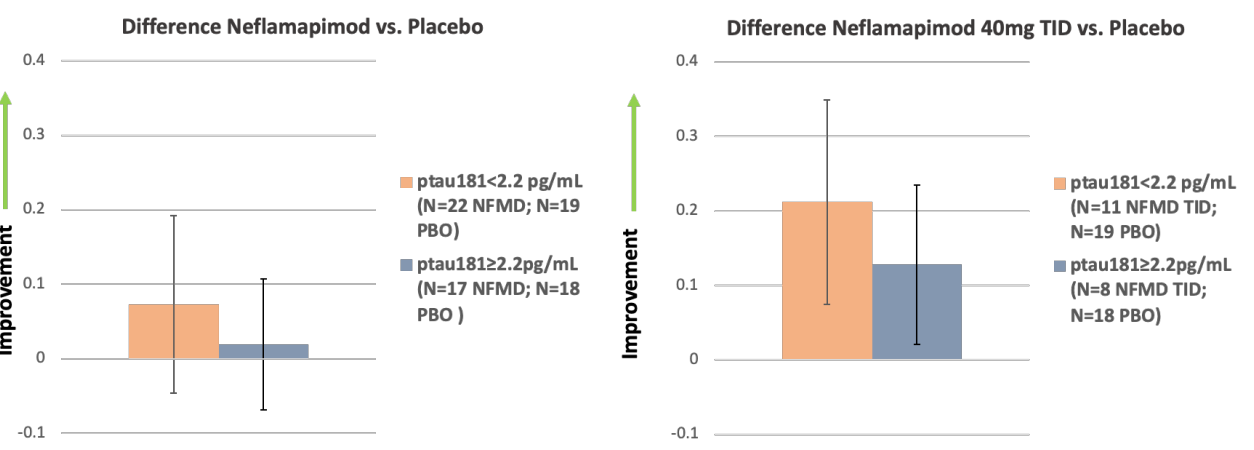

b. Attention Composite z-score

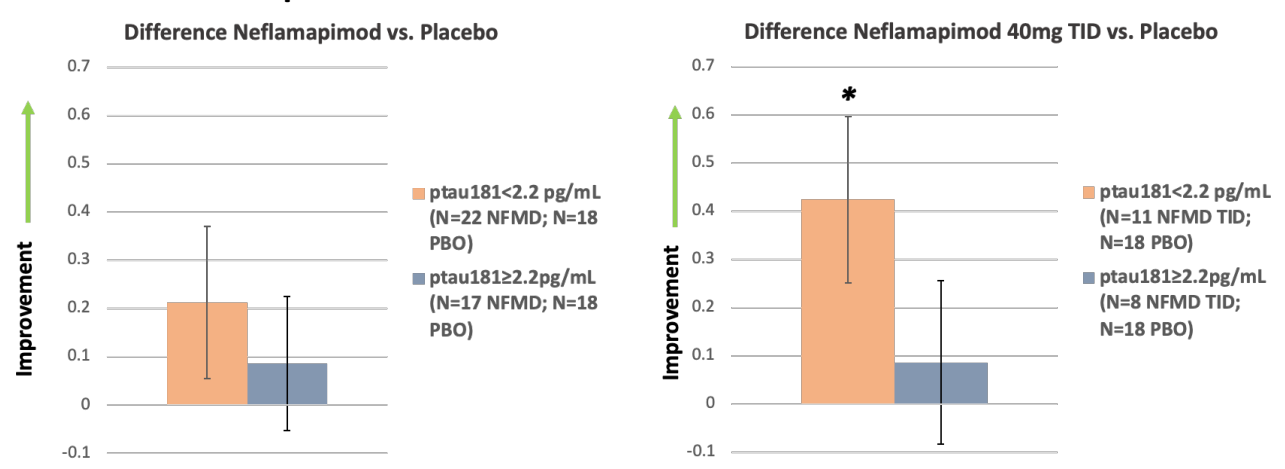

c. Clinical Dementia Rating Sum-of-boxes (CDR-SB)

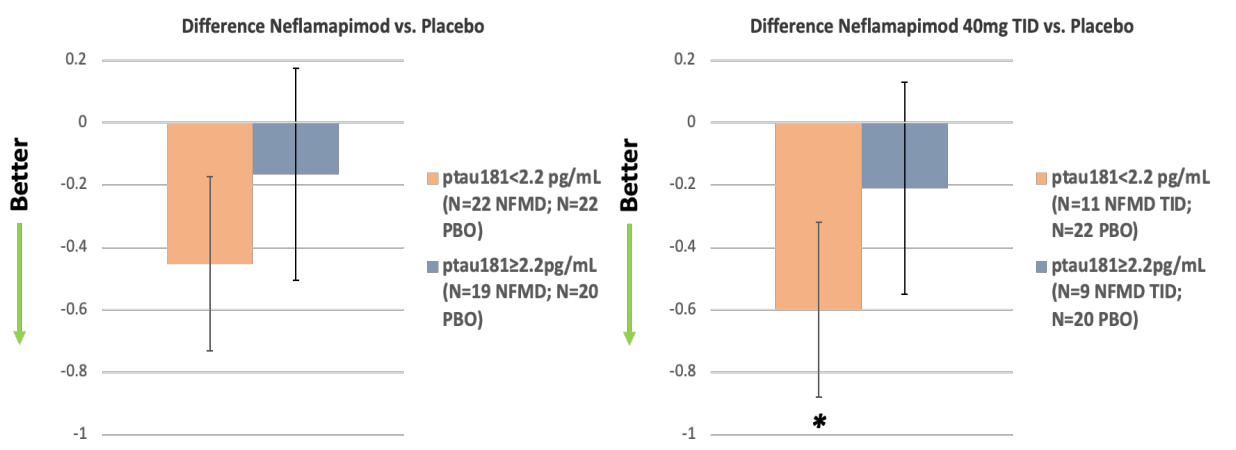

d. Time Up and Go (TUG) test
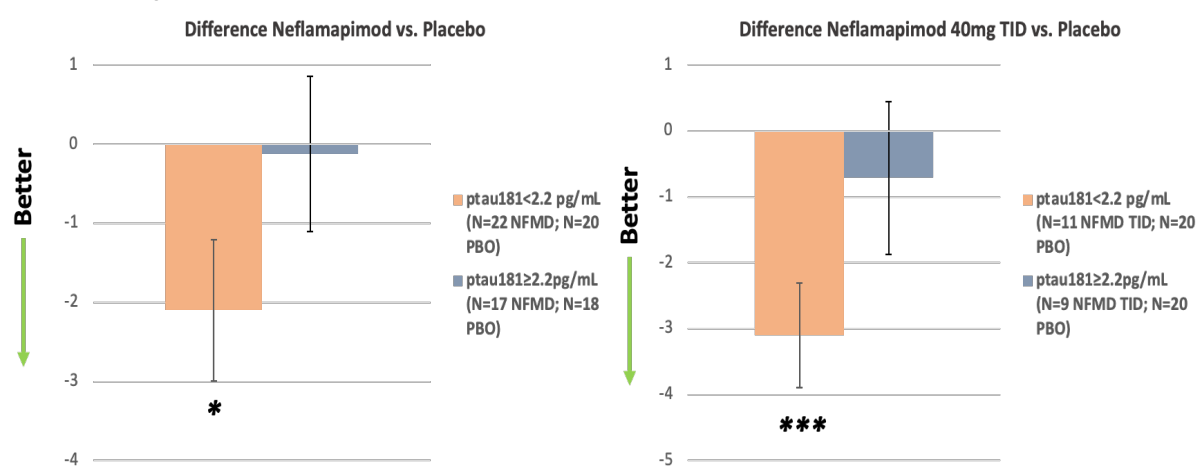


\section{Supplemental Figure 1. Consort Flow Diagram}

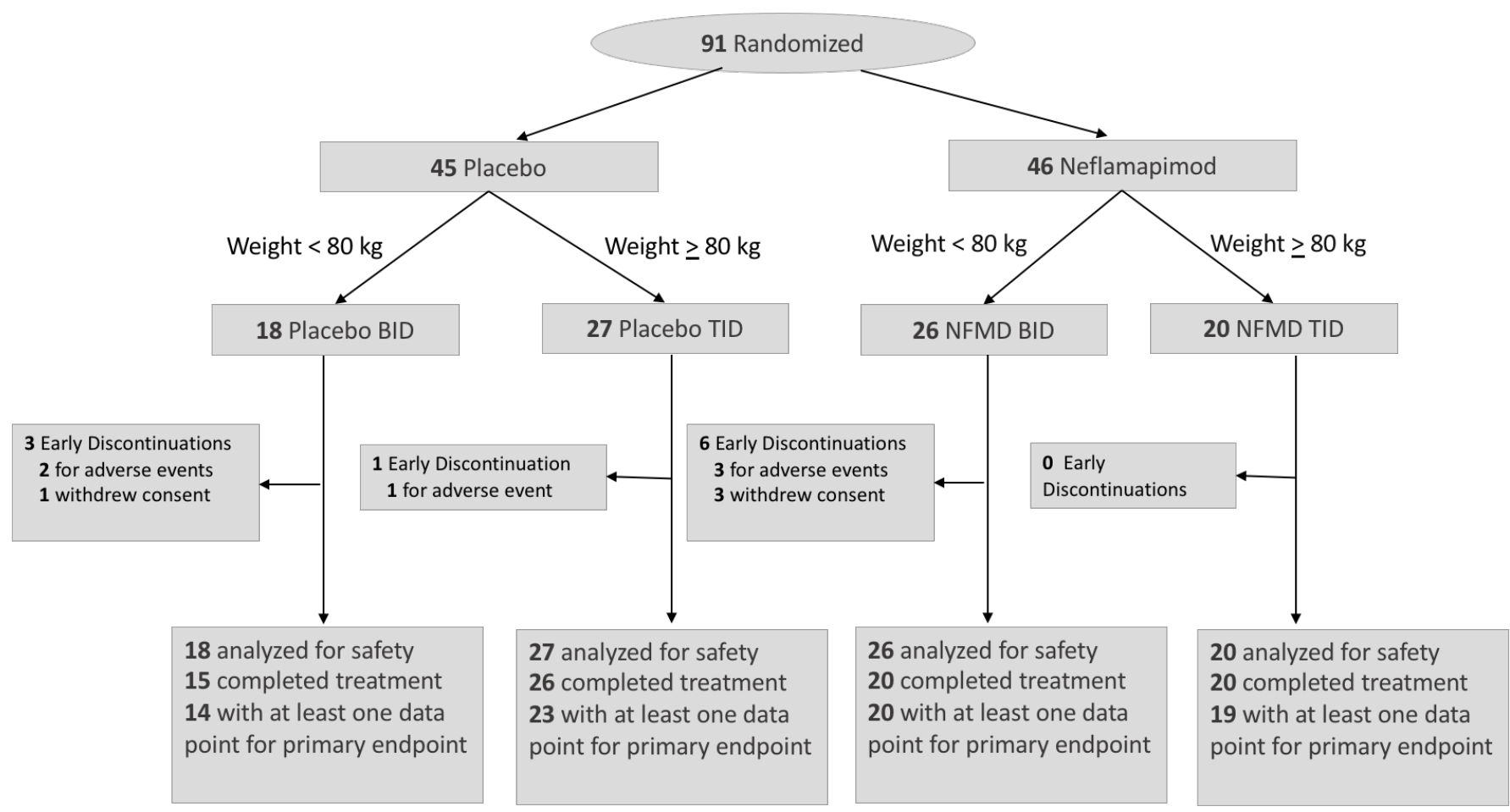


Supplemental Figure 2. Mean Change from Baseline in International Shopping List Test (ISLT)

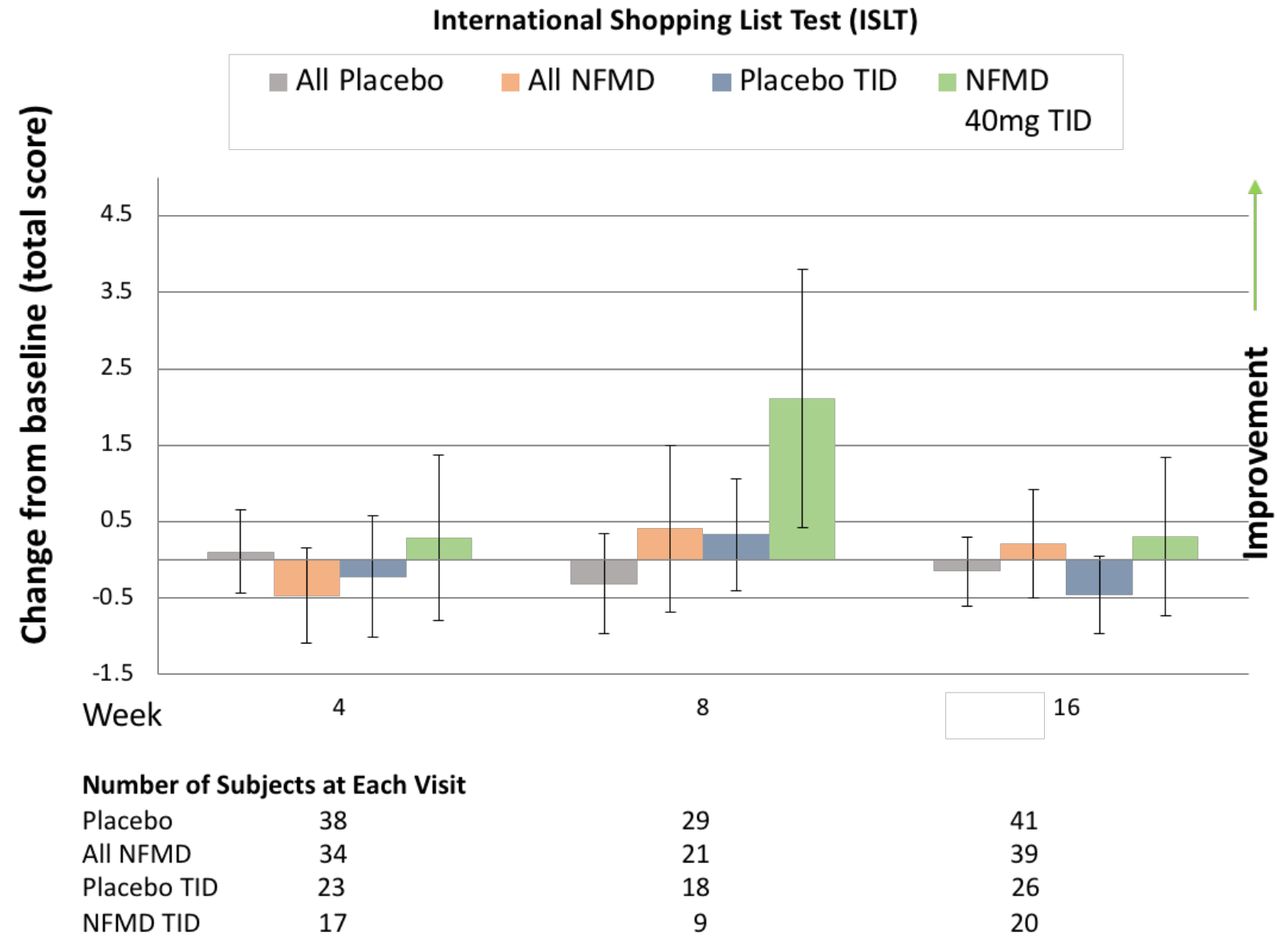


Supplemental Figure 3. Hallucinations Severity Over Time on Study

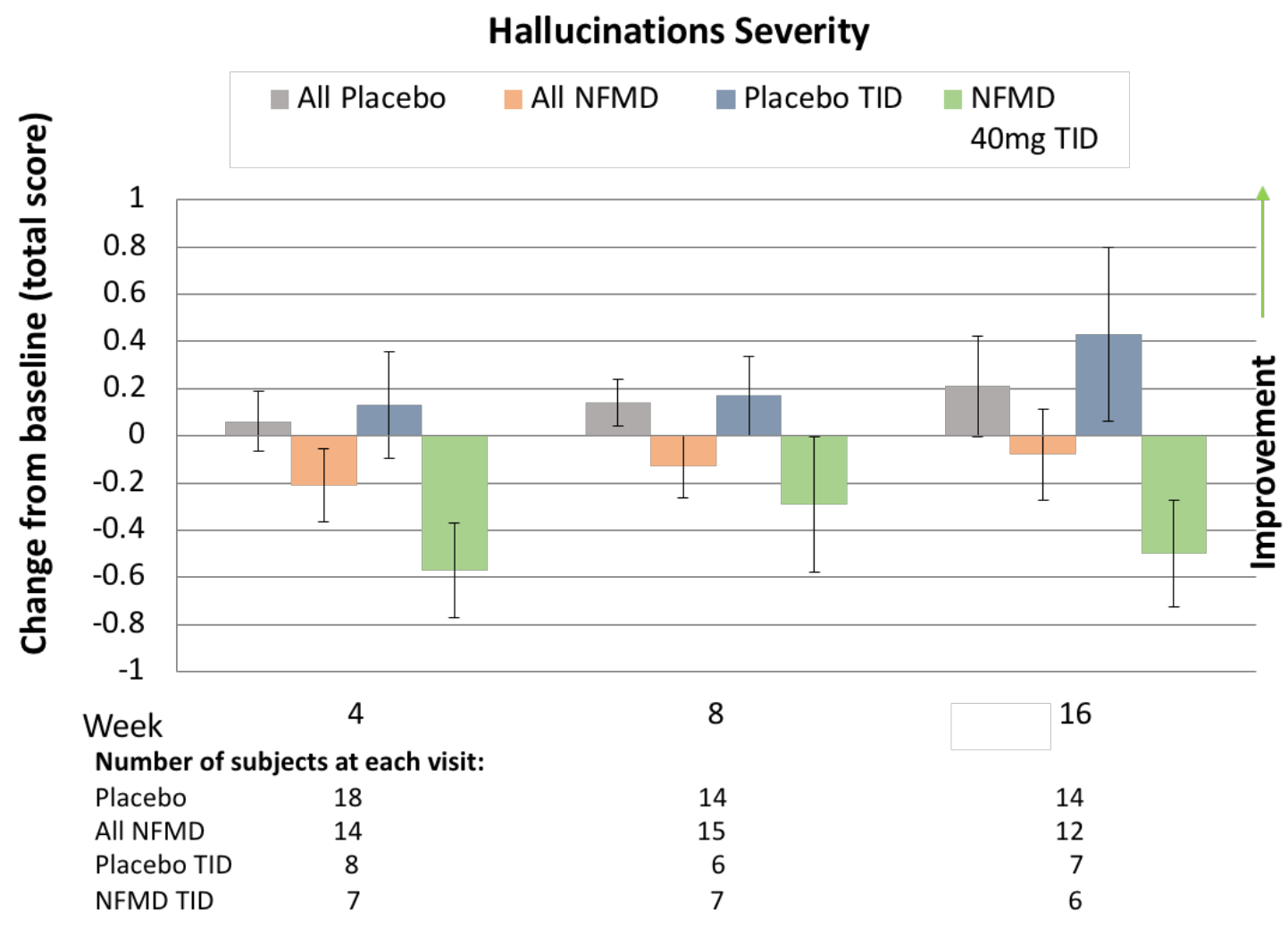




\section{Supplemental Figure 4. 10-Item Neuropsychiatric Inventory Domains of Interest}

\section{Hallucinations}

Hallucinations FxS Score
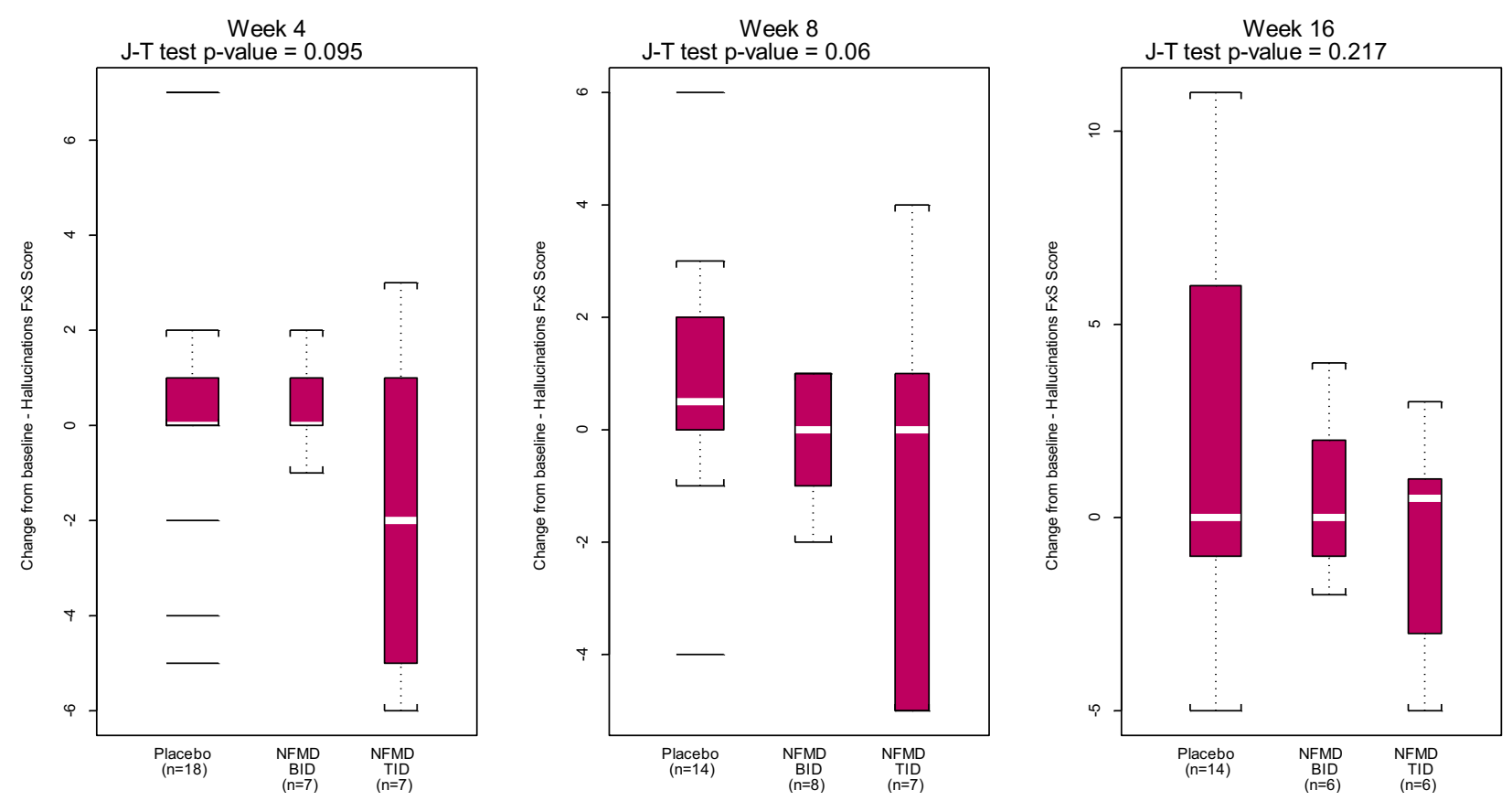

Agitation/Aggression

Agitation/Aggression FxS Score
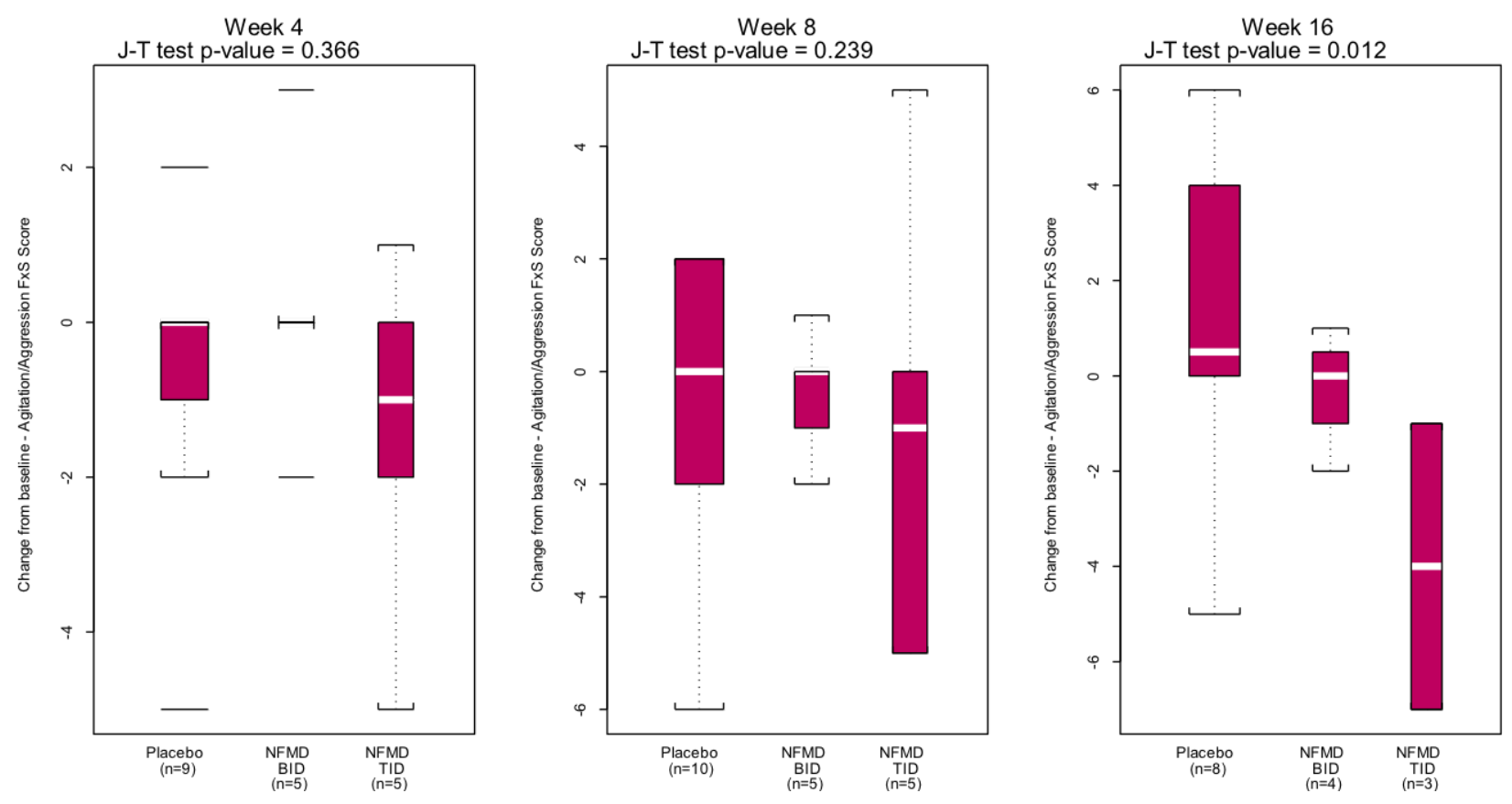
Depression/Dysphoria

Depression/Dysphoria FxS Score
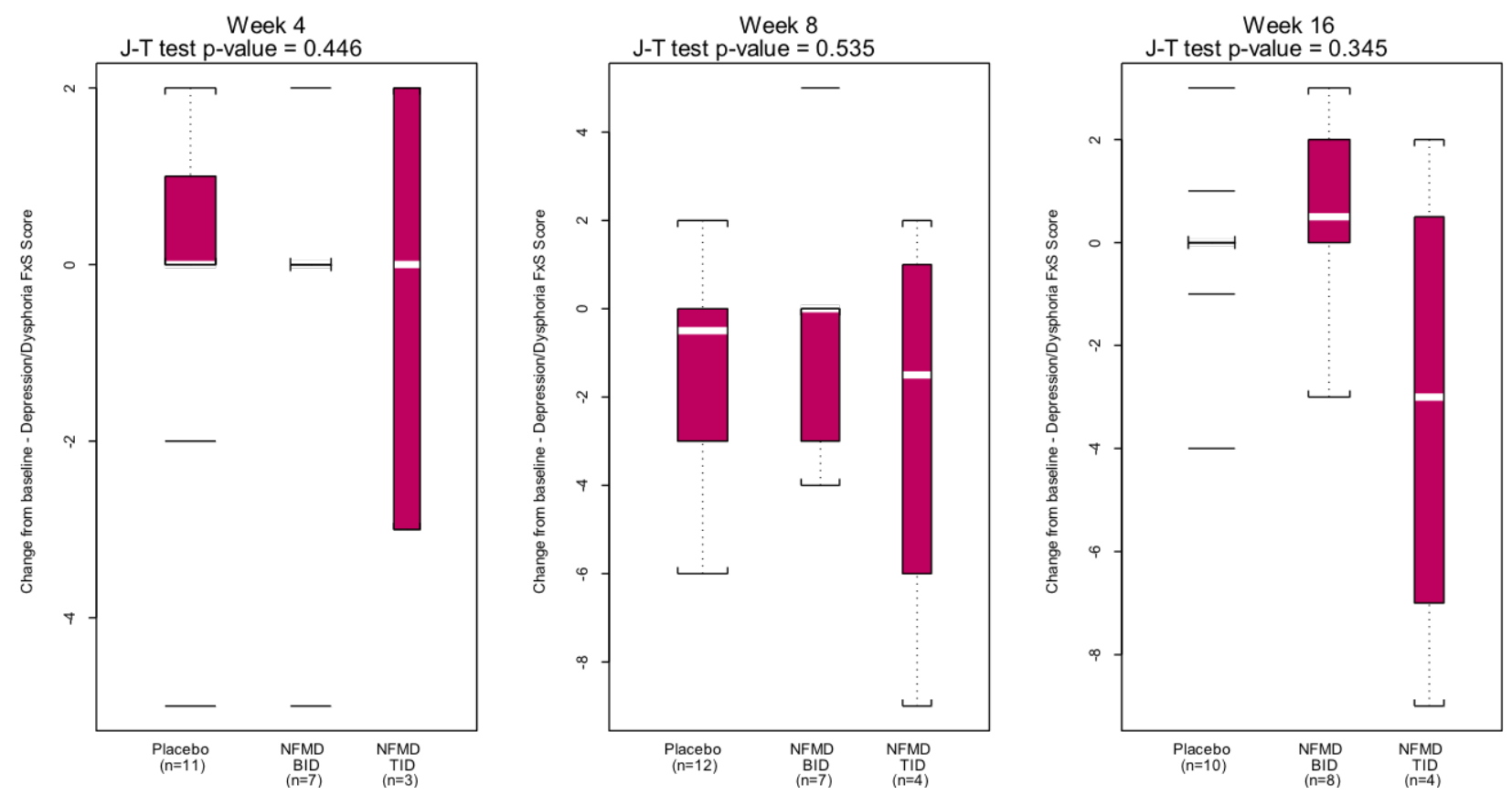

\section{Anxiety}
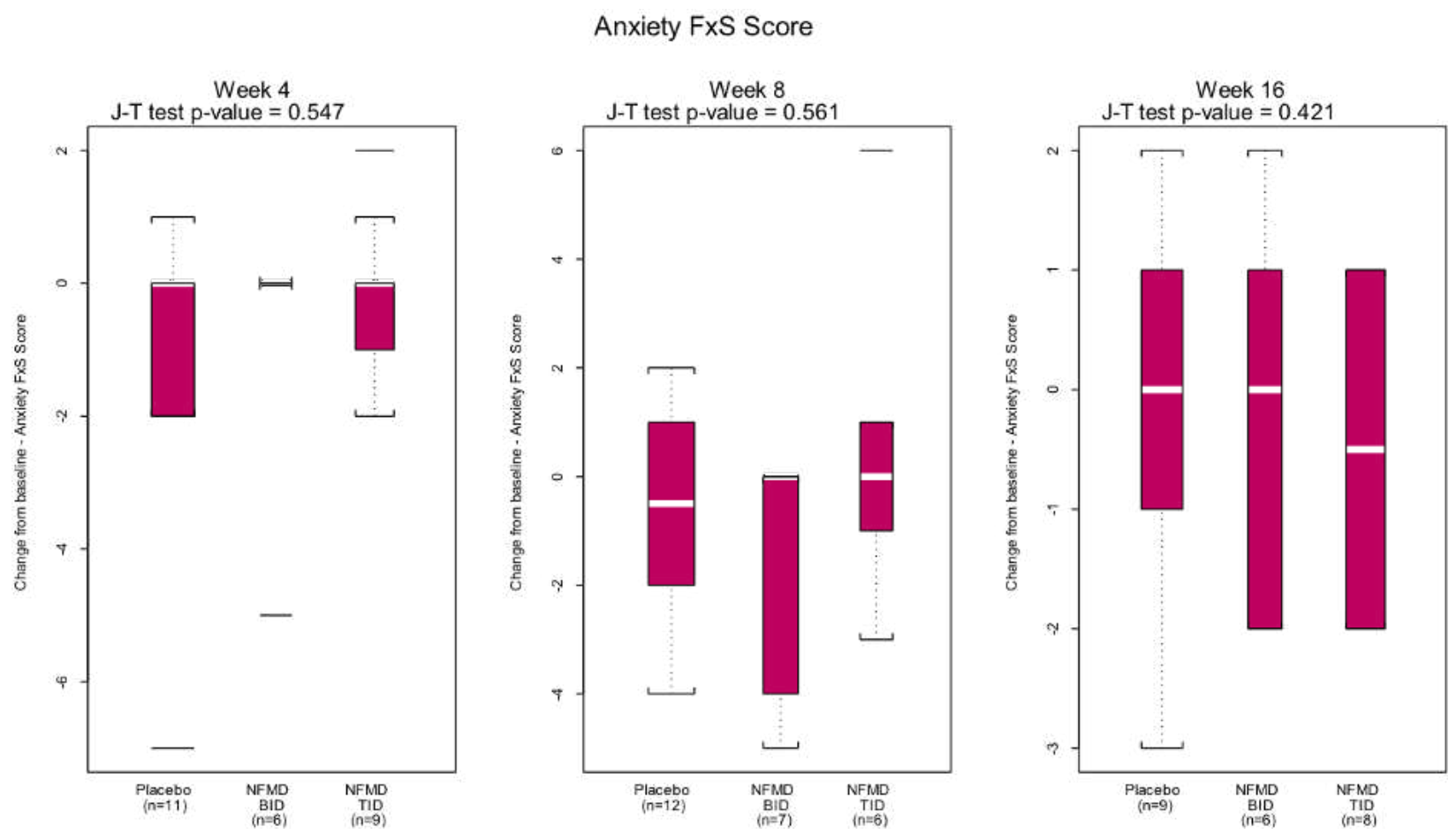
SUBMITTED MANUCSRIPT - CONFIDENTIAL

Supplemental Table 1. Baseline Disease Characteristics

\begin{tabular}{|c|c|c|c|c|}
\hline & $\begin{array}{l}\text { Placebo } \\
(\mathrm{N}=45)\end{array}$ & $\begin{array}{l}\text { NFMD ALL } \\
(\mathrm{N}=46)\end{array}$ & $\begin{array}{l}\text { Placebo TID } \\
(\mathrm{N}=27)\end{array}$ & $\begin{array}{l}\text { NFMD 40mg TID } \\
(\mathrm{N}=20)\end{array}$ \\
\hline Age (yrs) & $72.1(6.9)$ & $73.5(6.9)$ & $70.4(5.7)$ & $72.2(6.6)$ \\
\hline Male & $87 \%$ & $85 \%$ & $96 \%$ & $95 \%$ \\
\hline $\begin{array}{l}\text { CDR Sum of } \\
\text { Boxes }\end{array}$ & $5.1(3.2)$ & $4.9(1.8)$ & $4.3(2.3)$ & $4.7(1.8)$ \\
\hline MMSE & $23.0(3.3)$ & $23.1(3.9)$ & $23.6(3.3)$ & $23.4(3.3)$ \\
\hline $\begin{array}{l}\text { ISLT - } \\
\text { Immediate }\end{array}$ & $14.3(5.4)$ & $13.6(5.9)$ & $14.2(6.2)$ & $14.1(4.9)$ \\
\hline $\begin{array}{l}\text { ISLT - } \\
\text { Delayed }\end{array}$ & $4.3(2.1)$ & $3.9(2.7)$ & $4.3(2.4)$ & $4.2(2.4)$ \\
\hline $\begin{array}{l}\text { Timed Up } \\
\text { and Go } \\
\text { (seconds) }\end{array}$ & $13.5(6.4)$ & $12.7(3.7)$ & $13.3(5.2)$ & $13.3(3.8)$ \\
\hline
\end{tabular}




\section{Supplemental Table 2. Serious Adverse Events (SAEs) Reported in the Study and Discontinuations due to Treatment Emergent Adverse Events (TEAEs)}

\begin{tabular}{|l|c|l|}
\hline Treatment Group & $\begin{array}{c}\text { Number } \\
\text { of SAEs }\end{array}$ & Descriptions (All considered not related to study drug) \\
\hline Placebo BID & 3 & $\begin{array}{l}\text { Hematochezia on Day } 20 \\
\text { Internal Bleeding on Day } 72 \\
\text { Intraparenchymal hemorrhage on Day } 117\end{array}$ \\
\hline Placebo TID & 1 & Asthma exacerbation on Day 97 \\
\hline $\begin{array}{l}\text { Neflamapimod } \\
40 \mathrm{mg} \text { BID }\end{array}$ & 3 & $\begin{array}{l}\text { New brain lesions on Day } 84 \\
\text { Brain Tumor Diagnosis } 34 \text { days after last dose (Day 109) } \\
\text { Head Injury on Day } 19\end{array}$ \\
\hline $\begin{array}{l}\text { Neflamapimod } \\
40 \mathrm{mg} \text { TID }\end{array}$ & 0 & - \\
\hline
\end{tabular}

\section{Subjects Withdrawn due TEAE}

\begin{tabular}{|l|l|l|l|l|l|}
\hline & TEAE & $\begin{array}{l}\text { Study } \\
\text { Day }\end{array}$ & Severity & $\begin{array}{l}\text { Relationship to } \\
\text { Study Drug }\end{array}$ & Serious \\
\hline \multirow{3}{*}{ Neflamapimod 40mg BID } & Brain lesion & 84 & Severe & Not Related & Y \\
\cline { 2 - 6 } & Somnolence & 18 & Moderate & Possibly Related & N \\
\cline { 2 - 6 } & Head injury & 19 & Moderate & Not Related & Y \\
\hline Placebo BID & Hematochezia & 20 & Moderate & Not Related & Y \\
\hline
\end{tabular}




\section{Supplementary Files}

This is a list of supplementary files associated with this preprint. Click to download.

- flatAlamepc.pdf

- flatAlamrs.pdf 